\title{
SUPERSYMMETRY IN EUCLIDEAN QUANTUM FIELD THEORY
}

\section{Z. Haba}

Institut of Theoretical Physics

University of Wroclaw, Wroclaw, Poland

\section{J. Kupsch}

Fachbereich Physik

Universität Kaiserslautern, Kaiserslautern, Germany

Supersymmetry is formulated within a functional approach of euclidean quantum field theory. The rôle of the euclidean Lie superalgebra and its relation to the Poincare Lie superalgebra are investigated in detail. As example we study the Wess-Zumino model in two dimensions. 



\section{Introduction}

Supersymmetry of a relativistic quantum field theoretical model is usually based on the supersymmetry of a classical Lagrangean (with anticommuting fermi fields) and on a (formal) representation of the Poincaré Lie superalgebra on the physical state space, see e.g. $[37,40,43,46]$. These prerequesites are sufficient to derive a perturbation expansion with supersymmetric Ward identities for the time ordered Green's functions in a Schwinger-Symanzik type formalism of the generating functional or with a Berezin type of integration, see e.g. $[14,37]$. On the other hand for functional integration the euclidean approach is more adequate. A euclidean formulation of the Wess-Zumino (WZ) model in four dimensions has been given by Nicolai [32], and a functional version of this model in two dimensions based on euclidean superfields can be found in the lectures of Jaffe and Lesniewski [19]. But these approaches do not incorporate the rôle of the euclidean Lie superalgebra which has been studied e.g. in $[27,28]$. The significance of this algebra and the type of its representation in euclidean quantum field theory is not clarified in the literature [29].

In this paper we present a general definition of euclidean supersymmetry which incorporates the euclidean Lie superalgebra and which treats bosons and fermions with the same techniques of tensor algebras. To avoid all problems with euclidean field operators or with stochastic fields for fermions we use the functional point of view which goes back to Osterwalder and Schrader [34] in a formulation related to the Wightman functional of Borchers [2]. The generating functional of all Schwinger functions is then a linear functional on the free tensor algebra $T(\mathcal{S})$ of test functions of a space $S=S\left(\mathbb{R}^{\mathrm{d}}\right) \oplus \mathbf{C}^{\mathrm{N}}$. The symmetry properties (either totally symmetric or totally antisymmetric) of the Schwinger functions yield an ideal $J$ of equivalent test functions. The factor algebra $\mathcal{A}(\mathcal{S})=I / \mathcal{J}$ is a $\mathbb{I}_{2}$ graded algebra or superalgebra which is isomorphic to $\mathcal{A}^{+}\left(\mathcal{S}_{\mathrm{B}}\right) \bullet \mathcal{K}\left(\mathcal{S}_{\mathrm{F}}\right)$. Here $\mathcal{S}_{\mathrm{B}}$ and $\mathcal{S}_{\mathrm{F}}$ are the basic test function spaces for the bosonic or fermionic fields, $\mathcal{S}=\mathcal{S}_{\mathrm{B}} \oplus \mathcal{S}_{\mathrm{F}}$, and $\mathcal{A}^{\epsilon}(\boldsymbol{X})$ denotes for $\epsilon=+1$ the algebra of symmetric tensors and for $\epsilon=-1$ the algebra of antisymmetric tensors (Grassmann 
algebra) of the space $\boldsymbol{x}$. There is an abundand literature about graded algebras (superalgebras), graded manifolds and superanalysis, the list $[4,7,15,20,26,38,39,41$, $42,44]$ is not nearly complete. Nevertheless we shall present the mathematics of the superalgebra of test functions in some detail in Sect. 2,, because the cited literature is either too general or it elaborates on superspaces (mainly based on Grassmann algebras) which are not adequate for our construction.

In Sect. 3 the generating functional of all Schwinger functions is presented as linear functional $\mathrm{S}$ on the test function algebra $\mathcal{\Lambda}(\mathcal{S}): \mathrm{F} \in \mathcal{\Lambda}(\mathcal{S}) \rightarrow\langle\mathrm{S} \mid \mathrm{F}\rangle \in \mathrm{C}$, such that the restriction to $l_{n}$, the space of all tensors of rank $n$, exactly yields the $n$-point Schwinger functions. Then Osterwalder-Schrader (OS) positivity, euclidean invariance and supersymmetry are formulated for these functionals. The investigation of supersymmetry starts with a discussion of the complex euclidean Lie superalgebra which includes the real Lie algebra of the euclidean transformations. The complex euclidean Lie superalgebra $\mathcal{E}^{C}$ is represented by differential operators $L$ on the basic test function space $\mathcal{S}$. The corresponding superderivations $\mathrm{D}_{\mathrm{L}}, \mathrm{L} \in \mathcal{E}^{\mathrm{C}}$, provide then a representation of that Lie superalgebra on the whole algebra of test functions $\mathcal{\Lambda}(\mathcal{S})$. A theory with generating functional $S$ is supersymmetric if $\left\langle S \mid D_{L} F\right\rangle=0$ for all test functions $F \in \mathcal{A}(\mathcal{S})$ and all superderivations $\mathrm{D}_{\mathrm{L}}, \mathrm{L} \in \mathcal{E}^{\mathbb{C}}$. The representation of the complex euclidean Lie superalgebra is continuous in the nuclear topology of the test function algebra. All efforts to impose a reality/hermiticity condition on the representation leads to serious difficulties which can be overcome only by modifying the algebra [29]. Hermiticity with respect to the OS form singles out the real Poincaré Lie superalgebra and ensures supersymmetry of the reconstructed minkowskian theory.

Supersymmetry is easier formulated with auxiliary bosonic degrees of freedom. Also in our approach the auxiliary degrees of freedom are necessary for a simple linear representation of the Lie superalgebra. The corresponding Schwinger functions cannot 
be derived from a nonnegative bosonic measure. But all "unphysical" features disappear in the nontrivial kernel of the OS form if one goes over to the reconstructed minkowskian theory. There is no need to modify the theory by introducing imaginary auxiliary fields as was done by Nicolai [32].

In the literature the euclidean quantum field theory for Majorana spinors is mainly based on the construction of field operators $[10,32,3]$. Here we shall follow a purely functional point of view which avoids the problems with euclidean field operators or with fermionic stochastic fields $[10,24]$. The generating functional for the Schwinger functions of massive Majorana fermions can be defined for $d=2,3,4 \bmod 8$ dimensions, i.e. exactly when minkowskian Majorana spinors exist [25]. For these dimensions a real unitary charge conjugation matrix $\mathrm{C}$ can be defined which satisfies the conditions

$$
\mathrm{C}^{\mathrm{T}}=-\mathrm{C} \text { and } \mathrm{C} \gamma_{\mu} \mathrm{C}^{\mathrm{T}}=-\gamma_{\mu}^{\mathrm{T}}
$$

for all hermitean Dirac matrices $\gamma_{\mu}, \mu=1, \ldots, \mathrm{d}, \gamma_{\mu} \gamma_{\nu}+\gamma_{\nu} \gamma_{\mu}=2 \delta_{\mu \nu}$. The two point Schwinger function of the free Majorana field operator, i.e. the analytic continuation of $<\operatorname{vac}|\psi \psi| \mathrm{vac}>$, is given by $\mathrm{S}(\mathrm{x}, \mathrm{y}) \mathrm{C}$ with $\mathrm{S}(\mathrm{x}, \mathrm{y})=\left[\mathrm{m}-\sum_{\mu=1}^{\mathrm{d}} \gamma_{\mu} \partial_{\mu}\right]^{-1}(\mathrm{x}, \mathrm{y})=$ $(m-\not \partial)^{-1}(x, y)$. In writing formal euclidean lagrangeans we may therefore substitute the adjoint Majorana spinor $\tilde{\psi}_{\alpha}(x)$ by $\underset{\beta}{\Sigma} \mathrm{C}_{\alpha \beta} \psi_{\beta}(\mathrm{x})$ as in the minkowskian case and in agreement with the operator construction in [32].

In Sect. 4 we study as example the neutral WZ model in $\mathrm{d}=2$ dimensions. The formal euclidean lagrangean of this model is, see $[47,5,31]$ for the minkowskian version, 


$$
\begin{aligned}
& \mathcal{L}=\mathcal{L}_{\text {free }}+\mathcal{L}_{\text {int }} \\
& \begin{aligned}
\mathcal{L}_{\text {free }} & =-\frac{1}{2} \mathrm{~A}(\mathrm{x}) \Delta \mathrm{A}(\mathrm{x})-\frac{1}{2} \mathrm{~B}^{2}(\mathrm{x})+\mathrm{mA}(\mathrm{x}) \mathrm{B}(\mathrm{x})-\frac{1}{2} \psi^{\mathrm{T}}(\mathrm{x}) \mathrm{C}(\mathrm{m}-\not) \psi(x) \\
& =\frac{1}{2}(\mathrm{~A}(\mathrm{x}), \mathrm{B}(\mathrm{x}))\left[\begin{array}{rr}
-\Delta & \mathrm{m} \\
\mathrm{m} & -1
\end{array}\right]\left[\begin{array}{l}
\mathrm{A}(\mathrm{x}) \\
\mathrm{B}(\mathrm{x})
\end{array}\right]+\frac{1}{2} \psi^{\mathrm{T}}(\mathrm{x}) \mathrm{C}^{\mathrm{T}}(\mathrm{m}-\not \partial) \psi(\mathrm{x})
\end{aligned} \\
& \mathcal{L}_{\text {int }}=\lambda \mathrm{A}^{2}(\mathrm{x}) \mathrm{B}(\mathrm{x})-\lambda \psi^{\mathrm{T}}(\mathrm{x}) \mathrm{C} \psi(\mathrm{x}) \mathrm{A}(\mathrm{x}) .
\end{aligned}
$$

Here $\mathrm{A}$ and $\mathrm{B}$ are bosonic fields and $\psi(\mathrm{x})$ is a two component anticommuting Majorana spinor. Our formalism is used to study supersymmetry of the free WZ model and of perturbation theory. Moreover we indicate how the auxiliary degrees of freedom can be eliminated, and finally the generating functional is transferred to functional integrals of a type investigated by Jaffe et al. [17] and by Arai [1] in their hamiltonian solution of the WZ model.

In Appendix A we give some norm estimates for the algebra of test functions. In Appendix B we discuss the two point Schwinger functions of free charged or neutral spin -0 or spin- $\frac{1}{2}$ particles including the problem of OS positivity. Some emphasis is laid on a construction to derive the functionals for neutral particles from those of the charged ones. Appendix $\mathrm{C}$ presents some formulas which are needed for the calculation of euclidean functionals for Majorana fermions. 


\section{Graded Algebras}

In this section we introduce the basic $I_{2}$ graded algebra (superalgebra) of test functions, its topology and its dual algebra. The main references are $[4,41]$ and, concerning the topological structure, [13].

\subsection{Algebras of Test Functions}

Test functions and Schwinger functions are defined on $\Xi=\mathfrak{A} \times \mathbb{R}^{\mathrm{d}}$ where $\mathfrak{A}$ is a finite set with $|\mathfrak{A}|$ elements which label spin and field indices. The basic function space is now the complex linear space $\mathcal{S}(\Xi)$ of rapidly decreasing $\mathrm{C}^{\infty}$-functions. This space is a dense subspace of the Hilbert space $x=\mathcal{L}^{2}(\Xi)$ with the inner product

$$
(f \mid g)=\int f(\xi) g(\xi) d \xi=\underset{\alpha}{\Sigma} f(\alpha, x) g(\alpha, x) d x
$$

where $\xi=(\alpha, x) \in \Xi$, and the measure $d \xi$ includes Lebesgue integration with respect to $x \in \mathbb{R}^{d}$ and summation over $\alpha \in \mathfrak{A}$. The norm is denoted by $\|f\|$. Finer topologies can be defined with the differential operator of the shifted harmonic oszillator

$$
W f(\alpha, x)=\left(1+x^{2}-\Delta\right) f(\alpha, x)
$$

with $\mathrm{x}^{2}=\sum_{\mu=1}^{\mathrm{d}} \mathrm{x}_{\mu}^{2}$ and $\Delta=\sum_{\mu=1}^{\mathrm{d}} \frac{\partial^{2}}{\partial \mathrm{x}_{\mu}^{2}}$. This operator has been extensively used in white noise calculus [13]. This operator satisfies $W \geq \frac{2+d}{2}$ and $W^{-1}$ is a HilbertSchmidt operator on $x$. Then $\|\mathrm{f}\|_{\mathrm{p}}=\left\|\mathrm{W}^{\mathrm{p}} \mathrm{f}\right\|, \mathrm{p} \in \mathbb{I}$ is a family of norms on $\mathcal{S}(\Xi)$ which satisfy $\|\mathrm{f}\|_{\mathrm{p}} \leq\|\mathrm{f}\|_{\mathrm{p}+1}$. We denote by $\boldsymbol{x}_{(\mathrm{p})}=\mathrm{w}^{-\mathrm{p}} \boldsymbol{x}$ the closure of $\mathcal{S}$ with the norm $\|\mathrm{f}\|_{\mathrm{p}}$. The spaces $x_{(p)}$ are ordered by inclusion $x_{(p+1)} \subset x_{(p)}$, and $x_{(p)}$ is antidual to $x_{(-p)}$ with respect to the pairing (2.1). Since the embedding $x_{(p+1)} \subset x_{(p)}$ is given by the Hilbert-Schmidt operator $W^{-1}$ the intersection $\mathcal{S}(\Xi)=\prod_{\mathrm{p}=0}^{\infty} x_{(p)}$ is a nuclear space in the projective limit topology, see e.g. [11]. The antidual space is the space of tempered distributions $\mathcal{S}^{\prime}(\Xi)=\bigcup_{\mathrm{u}=0}^{\infty} x_{(-p)}$ 
To define a $\mathbb{Z}_{2}$ gradation the index set $\mathfrak{A}=\mathfrak{A}_{0} \cup \mathfrak{A}_{1}$ is split into nonempty subsets $\mathfrak{A}_{0}$ and $\mathfrak{A}_{1}, \mathfrak{A}_{0} \cap \mathfrak{A}_{1}=\emptyset$. The subspaces $\boldsymbol{X}_{\mathbf{k}} \subset \boldsymbol{X}$ of parity $\mathbf{k}=0,1$ are generated by functions $f(\alpha, x)$ which vanish on the complement of $\mathfrak{A}_{k}$, i.e. $f(\alpha, x)=0$ if $\alpha \in \mathfrak{A}_{k+1}$. Here and in the following $k+1$ is defined mod 2. The parity of a vector $f$ is denoted by $\pi(\mathrm{f}), \pi(\mathrm{f})=\mathbf{k}$ if $\mathrm{f} \in \boldsymbol{x}_{\overline{\mathrm{K}}}$. The respective test function spaces are $\mathcal{S}_{\overline{\mathrm{K}}}=\mathcal{S} \cap \boldsymbol{x}_{\overline{\mathbf{K}}}$.

For any involutive mapping $\alpha \rightarrow \alpha^{*}, \alpha^{* *}=\alpha$, on $\mathfrak{A}$ which does not mix the subsets $\mathfrak{A}_{\mathbf{k}}$, i.e. $\mathfrak{A}_{\mathbf{k}}^{*}=\mathfrak{A}_{\mathbf{k}}, \mathbf{k}=0,1$, we denote by $\xi=(\alpha, x) \rightarrow \xi^{*}=(\alpha, x)$ the corresponding transform on $\Xi$. Then

$$
f^{*}(\xi)=\overline{f\left(\xi^{*}\right)}
$$

is an antiunitary involution on $\boldsymbol{X}$ which preserves the parity. Moreover it is a continuous antilinear involution of the test function spaces $\mathcal{S}_{\mathbf{k}}, \mathrm{k}=0,1$. This involution yields a bilinear symmetric form $\langle\mathrm{f} \mid \mathrm{g}\rangle=\left(\mathrm{f}^{*} \mid \mathrm{g}\right)$ on $\boldsymbol{X}$, which is the duality pairing of $x_{(p)}$ and $x_{(-p)}$

The tensor space $\boldsymbol{X}^{\oplus \mathrm{n}}$ is identified with $\mathcal{L}^{2}\left(\Xi^{\mathrm{n}}\right)$. The tensor product of $\mathrm{F} \in \boldsymbol{X}^{\oplus \mathrm{m}}$ and $\mathrm{G} \in \boldsymbol{X}^{\mathrm{O}}$ is the numerical multiplication

$$
(\mathrm{F} \bullet \mathrm{G})\left(\xi^{1}, \ldots, \xi^{\mathrm{m}+\mathrm{n}}\right)=\mathrm{F}\left(\xi^{1}, \ldots, \xi^{\mathrm{m}}\right) \mathrm{G}\left(\xi^{\mathrm{m}+1}, \ldots, \xi^{\mathrm{m}+\mathrm{n}}\right)
$$

The Schwinger functions are totally symmetric in the bosonic arguments $\xi \in \Xi_{0}$ and totally antisymmetric in the fermionic arguments $\xi \in \Xi_{1}$. This type of mixed symmetry can be imposed on $\mathcal{L}^{2}\left(\Xi^{\mathrm{n}}\right)$ by

$$
\mathbf{P}_{\mathbf{n}} \mathbf{F}\left(\xi^{1}, \ldots, \xi^{\mathbf{n}}\right)=\frac{1}{\mathbf{n} !} \sum_{\sigma} \chi_{\sigma}\left(\alpha^{1}, \ldots, \alpha^{\mathbf{n}}\right) \mathbf{F}\left(\xi^{\sigma(1)}, \ldots, \xi^{\sigma(\mathbf{n})}\right)
$$

with the sign function $\chi_{\sigma}\left(\alpha^{1}, \ldots, \alpha^{\mathrm{n}}\right)=(-1)^{\mathrm{N}}$ where $\mathrm{N}_{\sigma}$ counts the inversions of fermionic arguments, $N_{\sigma}=\#\left\{(i, j) \mid i<j, \sigma(i)>\sigma(j), \alpha^{\sigma(i)} \in \mathfrak{A}_{1}, \alpha^{-(j)} \in \mathfrak{A}_{1}\right\}$. 
The symmetrized tensor space will be denoted by $\boldsymbol{l}_{\mathrm{n}}(\boldsymbol{x})=\mathrm{P}_{\mathrm{n}} \boldsymbol{x}^{\otimes \mathrm{n}}$. A tensor $\mathrm{F} \in \boldsymbol{l}_{\mathrm{n}}(\boldsymbol{x})$ has a defined parity $\pi(F)=k$ if $F\left(\alpha^{1}, x^{1}, \ldots, \alpha^{n}, x^{n}\right)=0$ for all $\left(\alpha^{1}, \ldots, \alpha^{n}\right) \in \mathfrak{A}^{n}$ with $\sum_{j=1}^{n} \alpha^{i} \neq k \bmod 2$ and arbitrary $x^{i} \in \mathbb{R}^{d}$. The graded tensor product of $F \in \lambda_{m}(x)$ and $\mathrm{G} \in \mathcal{h}_{\mathbf{n}}(\boldsymbol{x})$ is defined as

$$
F \circ G=\frac{(m+n) !}{m ! n !} P_{m+n} F \odot G
$$

which is an element of $\ell_{\mathrm{m}+\mathrm{n}}(x)$. The algebraic sum $\mathcal{A}(x)=\underset{\mathrm{n}=0}{\infty} \boldsymbol{l}_{\mathrm{n}}(x)$ is then a $\mathbb{u}_{2}$-graded algebra or superalgebra $[26,41]$ which is isomorphic to $\boldsymbol{l}^{+}\left(\boldsymbol{x}_{\overline{0}}\right) \bullet \kappa\left(\boldsymbol{x}_{\mathrm{T}}\right)$ where $\mathcal{A}^{+}\left(x_{\overline{0}}\right)$ is the algebra of symmetric tensors of $x_{\bar{\delta}}$ and $\mathcal{K}\left(x_{\bar{T}}\right)$ is the algebra of antisymmetric tensors of $\boldsymbol{x}_{\mathrm{T}}$. The graded tensor product satisfies the rule of a commutative superalgebra $F \circ G=(-1)^{\pi(F) \pi(G)} G \circ F$ for tensors with defined parity.

All definitions (2.4) $-(2.6)$ can be applied to test functions $\mathrm{F} \in \mathcal{S}\left(\Xi^{\mathrm{n}}\right) \subset \mathcal{L}^{2}\left(\Xi^{\mathrm{n}}\right)$ such that we can define the superalgebra $\mathcal{A}(\mathcal{S})$ of test functions in the same way.

A natural norm on $\boldsymbol{X}^{\text {n }}$ and consequently on $\mathcal{L}_{\mathrm{n}}(\boldsymbol{x})$ is the $\mathcal{L}^{2}\left(\Xi^{\mathrm{n}}\right)$ norm which we denote by $\|F\|$. On the algebra $\mathcal{A}(\boldsymbol{x})$ we define a family of norms, indexed by $\gamma \in \mathbb{R}$,

$$
\|\mathrm{F}\|_{\gamma}^{2}=\sum_{\mathrm{n}=0}^{\infty}(\mathrm{n} !)^{\gamma-1}\left\|\mathrm{~F}_{\mathrm{n}}\right\|^{2}
$$

if $F=\sum_{n=0}^{\infty} F_{n}, F_{n} \in \Lambda_{n}(x)$ and $\left\|F_{n}\right\|$ being the $\mathcal{L}^{2}\left(\Xi^{n}\right)$ norm. The closure of $\mathcal{A}(x)$ with this norm is the Fock space $\mathcal{F}_{(\gamma)}(\boldsymbol{X})$. The involution (2.3) on $\boldsymbol{X}$ is extended to an (antiunitary) involution on the algebras $\mathcal{A}(\mathcal{S})$ and $\mathcal{A}(\mathcal{X})$ and on the Fock space $\mathcal{F}_{(\gamma)}(\mathcal{X})$ by $\left.\mathbf{F}^{*}\left(\xi^{1}, \ldots, \xi^{\mathrm{n}}\right)=\overline{\mathrm{F}\left(\xi^{\mathrm{n}}, \ldots, \xi^{\mathrm{I}}\right.}\right)$ which satisfies the usual rule $(\mathrm{F} \circ \mathrm{G})^{*}=\mathrm{G}^{*} \circ \mathrm{F}^{*}$. The normalizations (2.6) and (2.7) yield the following identity for the norm 


$$
\left\|\mathrm{f}_{1} \circ \ldots \circ \mathrm{f}_{\mathbf{n}}\right\|_{\gamma}^{2}=(\mathrm{n} !)^{\gamma}{\underset{\sigma}{\Sigma}}_{\sigma}\left(\mathrm{f}_{1}, \ldots, \mathrm{f}_{\mathbf{n}}\right)\left(\mathrm{f}_{\sigma(1)} \mid \mathrm{f}_{1}\right) \ldots\left(\mathrm{f}_{\sigma(\mathbf{n})} \mid \mathrm{f}_{\mathbf{n}}\right)
$$

The sign function $\chi_{\sigma}$ depends on the parities $\pi\left(f_{j}\right)$ as $\chi_{\sigma}=(-1)^{N_{\sigma}}$ with $\mathbf{N}_{\sigma}\left(\mathrm{f}_{1}, \ldots, \mathrm{f}_{\mathrm{n}}\right)=\#\left\{(\mathrm{i}, \mathrm{j}) \mid \mathrm{i}<\mathrm{j}, \sigma(\mathrm{i})>\sigma(\mathrm{j}), \boldsymbol{\pi}\left(\mathrm{f}_{\mathrm{i}}\right)=\boldsymbol{\pi}\left(\mathrm{f}_{\mathrm{j}}\right)=1\right\}$

in agreement with the rules given for the sign function in eq. (2.5). If $\pi\left(f_{i}\right)=0$ for all $i=1, \ldots, n$ we get the usual bosonic result $\| f_{1} \circ \ldots$ of ${ }_{n} \|^{2}=(n !)^{\gamma} \operatorname{per}\left(f_{i} \mid f_{j}\right)$ and if $\pi\left(f_{i}\right)=1$ for all $i=1, \ldots, n$ we obtain $\left\|f_{1} \circ \ldots o f_{n}\right\|^{2}=(n !){ }^{\gamma} \operatorname{det}\left(f_{i} \mid f_{j}\right)$.

To specify test functions and distributions we introduce a two parameter family of norms $\|\mathrm{F}\|_{(\gamma, \mathrm{p})}=\left\|\Gamma\left(\mathrm{W}^{\mathrm{p}}\right) \mathrm{F}\right\|_{\gamma}$ on $\mathcal{A}(\mathcal{S})$ with the operator (2.2) $\mathrm{W}$ and with parameters $\gamma \in \mathbb{R}$ and $\mathrm{p} \in \mathbb{Z}$. The closure of $\mathcal{\mu}(\mathcal{S})$ with the norm $\|\mathrm{F}\|_{(\gamma, \mathrm{p})}$ is exactly the Fock

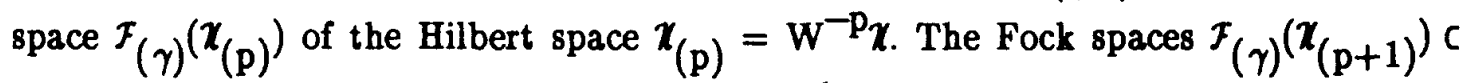
$\mathcal{F}_{(\gamma)}\left(\boldsymbol{x}_{(p)}\right)$ are imbedded by the operator $\Gamma\left(W^{-1}\right)$. In Appendix A we prove that this operator is a Hilbert-Schmidt operator on $\mathcal{F}_{(\gamma)}(\mathcal{X})$ for any $\gamma \in \mathbb{R}$. Hence the intersection $\mathcal{H}_{(\gamma)}(\mathcal{S}):=\bigcap_{\mathrm{p}=0}^{\infty} \mathcal{F}(\gamma)\left(\mathcal{X}_{(\mathrm{p})}\right)$ is a nuclear space [11]. For the main part of the paper it is sufficient to know that the graded tensor product (2.6) is defined within the algebra $\mathcal{A}(\mathcal{S})=\prod_{\mathrm{p}=0}^{\infty} \mathcal{A}\left(x_{(\mathrm{p})}\right)$. But this tensor product is continuous in the nuclear topology of the spaces $\lambda_{(\gamma)}(\mathcal{S})$ for any $\gamma \in \mathbb{R}$, the spaces $\lambda_{(\gamma)}(\mathcal{S})$ are therefore nuclear algebras.

If $T$ is a linear operator on $\boldsymbol{X}$ then the second quantization $\Gamma(T)$ is uniquely defined on $\mathcal{F}_{(\gamma)}(\boldsymbol{X})$ by the rule $\Gamma(\mathrm{T}) 1=1$ on the vacuum and by $\Gamma(\mathrm{T}) \mathrm{f}_{1} \circ \ldots$ of $\mathrm{o}_{\mathrm{n}}=$ $\left(\mathrm{Tf}_{1}\right) \circ \ldots \circ\left(\mathrm{Tf}_{\mathfrak{n}}\right)$ for arbitrary vectors $\mathrm{f}_{\mathrm{i}} \in \boldsymbol{X}$ and $\mathrm{n}=1,2, \ldots$. For the calculations of the subsequent sections two other types of linear operators on $\mathcal{A}(\boldsymbol{x})$ will be of special importance: derivations and antiderivations, see e.g. [4]. A linear operator D on $\mu(x)$ is called a derivation if it satisfies the Leibniz rule $D(F \circ G)=(D F) \circ G+F \circ(D G)$, it is called an antiderivation if it satisfies the modified Leibniz rule $D($ FoG $)=$ $(D F) \circ G+(-1)^{\pi(F)} F \circ(D G)$. There is a class of derivations and antiderivations which 
can be generated via the Leibniz rule from operators on the basic Hilbert space $\boldsymbol{X}$. Let $T$ be an even operator on $x$, i.e. an operator which does not change the degree, $\mathrm{T} \boldsymbol{x}_{\bar{\delta}} \subset \boldsymbol{x}_{\bar{\delta}}$ and $\mathrm{T} \boldsymbol{x}_{\mathrm{T}} \subset \boldsymbol{x}_{\mathrm{T}}$, then the Leibniz rule generates a unique derivation $\mathrm{D}_{\mathrm{T}}^{+}$on $\mathcal{A}(\boldsymbol{x})$ which reduces to $\mathrm{T}$ on $\boldsymbol{x}$. On the other hand for any odd operator $\mathrm{A}$ on $\boldsymbol{x}$, i.e. an operator which changes the degree, $A x_{\delta} \subset x_{T}$ and $A x_{I} \subset x_{\bar{\sigma}}$, the modified Leibniz rule generates a unique antiderivation $D_{A}^{-}$on $\mathcal{A}(\boldsymbol{x})$ such that $D_{A}^{-} f=A f$ for $f \in \mathcal{X}$. The generalizations of these constructions are superderivations $[26,41]$. Any operator $T=\boldsymbol{X} \rightarrow \boldsymbol{X}$ can be split into an even and an odd part $T=T_{0}+T_{1}$ with $T_{i} \boldsymbol{x}_{\bar{k}} \subset \boldsymbol{x}_{\overline{i+k}}$, the index $i$ is called the parity of the operator. The linear operator on $\mathcal{A}(x)$

$$
\mathrm{D}_{\mathrm{T}}=\mathrm{D}_{\mathrm{T}_{0}}^{+}+\mathrm{D}_{\mathrm{T}_{1}}^{-}
$$

is called a superderivation. If $\mathrm{T}$ is continuous this superderivation can always be

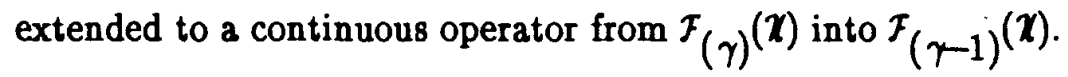

\subsection{Dual Algebras}

An essential problem of this paper is to evaluate linear functionals on the graded algebra $\mathcal{\Lambda}(\mathcal{S})$ or on a Fock space $\mathcal{F}_{(\gamma)}\left(\boldsymbol{x}_{(\mathrm{p})}\right)$. The duality pairing $\langle\mathrm{f} \mid \mathrm{g}\rangle=\left(\mathrm{f}^{*} \mid \mathrm{g}\right)$ between $x_{(-p)}$ and $x_{(p)}$ can be extended to a duality between the Fock spaces $\mathcal{F}_{(-\gamma)}\left(\boldsymbol{x}_{(-\mathrm{p})}\right)$ and $\mathcal{F}_{(\gamma)}\left(\boldsymbol{x}_{(\mathrm{p})}\right)$ by

$$
<f_{1} \circ \ldots \circ f_{n}\left|g_{1} \circ \ldots \circ g_{n}\right\rangle=\underset{\sigma}{\Sigma} \chi_{\sigma}\left(f_{1}, \ldots, f_{n}\right)<f_{\sigma(1)}\left|g_{1}\right\rangle \ldots<f_{\sigma(n)}\left|g_{n}\right\rangle
$$

with $f_{i} \in X_{(-p)}$ and $g_{j} \in X_{(p)}$. This duality respects the underlying algebraic structures, see e.g. [4] for the symmetric and the antisymmetric tensor algebras (the tensor product of which is isomorphic to the graded algebra considered here). The dual space of $\mathcal{l}_{(\gamma)}(\mathcal{S})$ is $\mathcal{l}_{(-\gamma)}\left(\mathcal{S}^{\prime}\right)={\underset{\mathrm{p}=0}{\bigcup} \mathcal{I}}_{(-\gamma)}\left(\boldsymbol{X}_{(-\mathrm{p})}\right)$. The norm estimates of Appendix A imply 
that the graded tensor product is continuous in the inductive limit topology of $\Lambda_{(-\gamma)}\left(\mathcal{S}^{\prime}\right)$

In the following sections we shall exploit the duality between the Fock spaces $\mathcal{F}_{(-\gamma)}\left(x_{(-\mathrm{p})}\right)$ and $\mathcal{F}_{(\gamma)}\left(\boldsymbol{X}_{(\mathrm{p})}\right)$ or between the algebras $\mathcal{A}_{(-\gamma)}\left(\mathcal{S}^{\prime}\right)$ and $\mathcal{A}_{(\gamma)}(\mathcal{S})$ to calculate linear functionals. Take as example a tensor $\Omega \in \mathcal{l}_{2}\left(x_{(-\mathrm{p})}\right)$. The first part of the norm estimate (A.3) implies that $\exp \Omega=\sum_{n=0}^{\infty} \frac{1}{n !} \Omega^{n}=1+\Omega+\frac{1}{2 !} \Omega \circ \Omega+.$. converges within $\mathcal{F}_{(\gamma)}\left(x_{(-p)}\right)$ if $\gamma<0$. Then for $f_{i} \in X_{(p)}, i=1, \ldots, 2 \mathrm{n}$,

$$
\begin{aligned}
& <\exp \Omega\left|\mathrm{f}_{1} \circ \ldots \circ f_{2 \mathrm{n}}\right\rangle= \\
& \frac{1}{2^{\mathrm{n}} \mathrm{n} !} \Sigma \chi_{\sigma}\left(\mathrm{f}_{1}, \ldots, \mathrm{f}_{2 \mathrm{n}}\right) \omega\left(\mathrm{f}_{\sigma(1)}, \mathrm{f}_{\sigma(2)}\right) \ldots \omega\left(\mathrm{f}_{\sigma(2 \mathrm{n}-1)}, \mathrm{f}_{\sigma(2 \mathrm{n})}\right)
\end{aligned}
$$

yields a gaussian combinatorics of the two point functions $\omega(f, g)=\langle\Omega \mid f \circ g\rangle$. The sign function $\chi_{\sigma}$ has already been used in (2.9). If $\pi\left(f_{i}\right)=0$ for all $i=1, \ldots, 2 n$ we get the usual bosonic result with the hafnian $<\exp \Omega \mid f_{1} \circ \ldots \circ f_{2 n}>=\operatorname{haf}\left(\omega\left(f_{i}, f_{j}\right)\right)$ and if $\pi\left(f_{i}\right)=1$ for $i=1, \ldots, 2 n$ we obtain a pfaffian $\langle\exp \Omega| f_{1} \circ \ldots \circ f_{2 n}>=p f\left(\omega\left(f_{i}, f_{j}\right)\right)$, see [6]

There is an important consequence of the duality (2.10) concerning superderivations (2.9). If $\mathrm{T}$ is a continuous linear operator on the test function space $\mathcal{S}$ then there exists a unique continuous operator $\mathrm{T}^{\prime}$ on $\mathcal{S}^{\prime}$ such that $\langle\mathrm{h} \mid \mathrm{Tf}\rangle=\left\langle\mathrm{T}^{\prime} \mathrm{h} \mid \mathrm{f}\right\rangle$ for $\mathrm{f} \in \mathcal{S}$ and $\mathrm{h} \in \mathcal{S}^{\prime}$. The duality (2.10) yields that the superderivations (2.9) $\mathrm{D}_{\mathrm{T}}$ and $\mathrm{D}_{\mathrm{T}^{\prime}}$ are again dual operators

$$
<\mathrm{D}_{\mathrm{T}^{\prime}}, \mathrm{H}|\mathrm{F}\rangle=\left\langle\mathrm{H} \mid \mathrm{D}_{\mathrm{T}} \mathrm{F}\right\rangle
$$


for arbitrary $F \in \mathcal{A}(\mathcal{S})$ and $H \in \mathcal{A}_{(-\gamma)}\left(\mathcal{S}^{\prime}\right), \gamma \in \mathbb{R}$. In the following sections we shall also use the interior product (contraction) $G\lrcorner F$ between $G \in \ell_{(-\gamma)}\left(\mathcal{S}^{\prime}\right)$ and $F \in \mathcal{A}_{(\gamma)}(S)$. This algebraic operation is defined by the identity

$$
\langle\mathrm{H} \circ \mathrm{G} \mid \mathrm{F}\rangle=\langle\mathrm{H} \mid \mathrm{G}-\mathrm{F}\rangle
$$

for arbitrary $\mathrm{H} \in \mathcal{A}_{(-\gamma)}(S)$, see [4].

\section{Schwinger Functions}

In the following we shall investigate a quantum field theory of spin-0 bosons and of spin $-\frac{1}{2}$ fermions in $d \geq 2$ dimensions. The extension to more general field theories is indicated but not given explicitly. By our choice of the test function algebra fields with intermediate statistics (in $d=2$ or 3 dimensions) are excluded.

\subsection{The Generating Funtional}

Let $f_{i} \in \mathcal{S}, i=1, \ldots, n$, be test functions and $S_{n}\left(\xi_{1}, \ldots, \xi_{n}\right)$ be the $n$-point Schwinger function. Then

$$
S_{n}\left[f_{1}, \ldots, f_{n}\right]=\int S_{n}\left(\xi^{1}, \ldots, \xi^{n}\right) f_{1}\left(\xi^{1}\right) \ldots f_{n}\left(\xi^{n}\right) d \xi
$$

is a continuous $n$-linear functional which has the symmetry properties

$$
\begin{aligned}
& S_{n}\left[f_{1}, \ldots, f_{n}\right]=(-1)^{\pi\left(f_{i}\right) \cdot \pi\left(f_{i+1}\right)} S_{n}\left[f_{1}, \ldots, f_{i+1}, f_{i}, \ldots, f_{n}\right] . \text { Hence it can be written as } \\
& S_{n}\left[f_{1}, \ldots, f_{n}\right]=\left\langle S_{n} \mid f_{1} \circ \ldots \circ f_{n}\right\rangle
\end{aligned}
$$

with the Schwinger function $S_{n}$ taken as element of $l_{n}\left(\mathcal{S}^{\prime}\right)$. For test functions $\mathrm{F}=\underset{\mathrm{n}}{\Sigma} \mathrm{F}_{\mathrm{n}} \in \mathcal{A}(\mathcal{S}), \mathrm{F}_{\mathrm{n}} \in \mathcal{l}_{\mathrm{n}}(\mathcal{S})$, the finite sum 


$$
\langle\mathrm{S} \mid \mathrm{F}\rangle=\underset{\mathrm{n} \geq 0}{\Sigma}\left\langle\mathrm{S}_{\mathbf{n}} \mid \mathrm{F}_{\mathbf{n}}\right\rangle
$$

with $S=\sum_{\mathfrak{n}=0}^{\infty} S_{\mathfrak{n}}, S_{0}=1$, is then the generating functional for all Schwinger functions. The usual assumptions about regularity and growth of Schwinger functions $[35,8]$ assure that $\langle\mathrm{S} \mid \mathrm{F}\rangle$ is continuous in a norm $\|\mathrm{F}\|_{(\gamma, \mathrm{p})}$ for some $\gamma \geq 0$ and some $\mathrm{p} \geq 0$. Hence $\mathrm{F} \in \Lambda_{(\gamma)}(\mathcal{S}) \longrightarrow<\mathrm{S} \mid \mathrm{F}>\in \mathrm{C}$ is continuous in the nuclear topology of $\lambda_{(\gamma)}(\mathcal{S})$.

For free fields the Schwinger functional $S$ is exactly the exponential of the two point function in the algebra $\mathcal{A}_{(-\gamma)}\left(\mathcal{S}^{\prime}\right)$. The correct gaussian combinatorics of the $\mathrm{n}$-point functions has already been seen in (2.11). It remains to discuss the two point function in more detail. For a theory with the minkowskian fields $\phi_{\alpha}(x), \alpha \in$ $\mathfrak{A}=\{1, \ldots, N\}, \underline{x} \in M^{d}$ beeing a Minkowski space variable, we have to choose test functions $f \in S(\Xi)=S\left(\mathbb{R}^{\mathrm{d}}\right) \bullet \mathrm{C}^{\mathrm{N}}$. The two point functions are obtained by analytic continuation of the matrix kernel $<\operatorname{vac}\left|\phi_{\alpha}(\underline{x}) \phi_{\beta}(y)\right| \operatorname{vac}>\rightarrow \Omega_{\alpha \beta}(x, y)$ to the euclidean domain $\mathbb{R}^{\mathrm{d}}$. The tensor of the two point functions determines then the bilinear form

$$
<\Omega \mid f \circ g>=\omega(f, g)=\underset{\alpha, \beta}{\Sigma} \int f(\alpha, x) \Omega_{\alpha^{*} \beta^{*}}(x, y) g(\beta, y) d x d y
$$

where $\alpha+\alpha^{*}$ is the involutive map of the index set $\mathfrak{A}$ which enters the involution (2.3). For neutral fields (particle $=$ antiparticle) we simply have $\alpha^{*}=\alpha$. For charged fields we shall take an index set $\mathfrak{A}=\{1, \ldots, 2 \mathrm{~N}\}$ such that $\alpha \in\{1, \ldots, N\} \rightarrow$ $\alpha+N \in\{N+1, \ldots, 2 N\}$ corresponds to the transition to the field of opposite charge (adjoint field). The involution will therefore be defined by $\alpha^{*}=\alpha+\mathrm{N} \bmod 2 \mathrm{~N}$.

For a free neutral spin zero field we need a one component test function $f \in \mathcal{S}\left(\mathbb{R}^{\mathrm{d}}\right)$, and the two-point Schwinger function is the inverse euclidean Klein-Gordon operator $D(x, y)=\left(m^{2}-\Delta\right)^{-1}(x, y)$. For a charged spin zero field $\phi(x), x \in M^{d}$, the kernel is calculated by analytic continuation of the matrix 


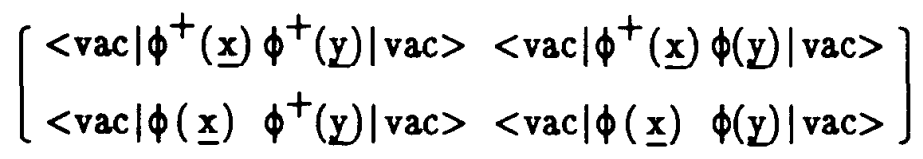

and the test function space has two components. The resulting symmetric form is then

$$
\omega(f, g)=\int f^{T}(x)\left[\begin{array}{cc}
0 & D(y, x) \\
D(x, y) & 0
\end{array}\right] g(y) d x d y .
$$

The Dirac spinor in $d=2,3, \ldots$ dimensions has $N_{d}=2^{[d / 2]}$ components. Hence we need test functions $f$ with $2 N_{d}$ components. Writing $f$ as column vector the bilinear skew symmetric form (3.4) for the Dirac field is [22,25]

$$
\omega_{D}(f, g)=\int f^{T}(x) \mu_{-}(x, y) g(y) d x d y
$$

with the matrix $u_{-}(x, y)=\left[\begin{array}{cc}0 & -S^{T}(y, x) \\ S(x, y) & 0\end{array}\right]$ where $S(x, y)$ is the kernel of the inverse euclidean Dirac operator $\mathrm{m}-\partial=\mathrm{m}-\Sigma_{\mu} \gamma_{\mu} \partial_{\mu}$.

In the literature there has been some discussion about the existence of euclidean Majorana fields, often based on an investigation of classical fields, see e.g. $[33,48]$. But euclidean classical fields are not necessary for the corresponding euclidean quantum field theory. Wightman functions and consequently Schwinger functions of Majorana fields exist if minkowskian field operators exist, i.e. for $d=2,3,4 \bmod 8$ dimensions. In these cases a real unitary charge conjugation matrix $\mathrm{C}$ can be defined which satisfies the conditions (1.1). The minkowskian Majorana field operator $\psi_{\alpha}(\underline{x})$ is related to the adjoint operator by $\tilde{\psi}_{\alpha}=\sum_{\beta} \mathrm{C}_{\alpha \beta} \psi_{\beta}$ and the Schwinger function of

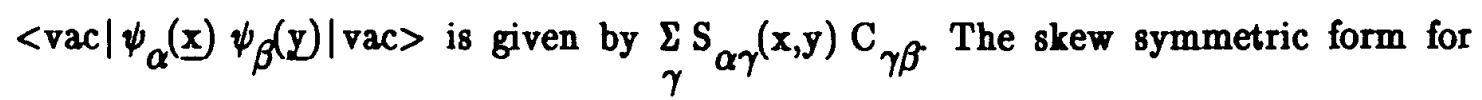
massive Majorana fields is therefore 


$$
\omega_{M}(f, g)=\int f^{T}(x) \mu(x, y) g(y) d x d y \quad \text { with } \mu(x, y)=S(x, y) C
$$

with $\mathrm{N}_{\mathrm{d}}$-component test functions. In the massless case another type of Majorana forms is also possible which we shall not use here, see [25]. More details about Schwinger functions of charged and neutral fields and relations between them are given in Appendix B.

Finally we present the bilinear form (3.4) for a theory of neutral spin zero bosons and of Majorana fermions as in the case of the neutral Wess-Zumino model $[45,46,47]$. In $d$ dimensions the Majorana spinor has $\mathbf{N}=\mathbf{N}_{\mathrm{d}}$ components. We shall assume already here that the bosonic sector has also $\mathrm{N}$ components, i.e. includes auxiliary fields. That assumption will be used in the sections about supersymmetry, but it is convenient to introduce the corresponding notations already here. The structure of the test function space $\mathcal{S}=\mathcal{S}\left(\mathbb{R}^{\mathrm{d}}\right) \bullet \mathrm{C}^{2 \mathrm{~N}}$ is chosen as $\mathcal{S}=\left[\begin{array}{l}\mathcal{S}_{\mathrm{B}} \\ \mathcal{S}_{\mathrm{F}}\end{array}\right]$ with $\mathcal{S}_{\mathrm{B}} \simeq \mathcal{S}_{\mathrm{F}} \simeq \mathcal{S}\left(\mathbb{R}^{\mathrm{d}}\right) \bullet \mathrm{C}^{\mathrm{N}}$. Here $\mathcal{S}_{\mathrm{B}}$ refers to bosonic, and $\mathcal{S}_{\mathrm{F}}$ to fermionic test functions. The bilinear form (3.4) reads in this notation

$$
\omega(f, g)=\int f^{T}(x) K(x, y) g(y) d x d y \quad \text { with } K(x, y)=\left[\begin{array}{cc}
\mathcal{D}(x, y) & 0 \\
0 & \mu(x, y)
\end{array}\right]
$$

The kernel for the bosons is a symmetric $N \times N$ matrix $\mathcal{D}(x, y)=\mathcal{D}(y, x)=\mathcal{D}^{T}(x, y)$. Here we only give the explicit form of this matrix kernel for $d=2$ dimensions with $N=2$. Then the bosonic sector of the WZ model includes a propagating spin zero field $A(x)$ and an auxiliary field $B(x)[47,3]$. The euclidean version of the free bosonic lagrangean has been given in the Introduction. The differential operator for the bosonic part is $\left[\begin{array}{cc}-\Delta & m \\ m & -1\end{array}\right]$, see (1.2). The inverse of this operator is $D(x, y)=\left[\begin{array}{cc}1 & m \\ m & \Delta\end{array}\right] D(x, y)$ with the Klein-Gordon propagator $D(x, y)=\left(m^{2}-\Delta\right)^{-1}(x, y)$. This matrix can be decomposed into

$$
D(x, y)=\left[\begin{array}{ll}
1 & m \\
m & m^{2}
\end{array}\right] D(x, y)-\left[\begin{array}{ll}
0 & 0 \\
0 & 1
\end{array}\right] \delta^{2}(x-y)
$$


The first term is a positive semidefinite kernel which corresponds to the propagation of a single spin zero field, since the matrix has rank one. The second term looks like a nonpropagating white noise, but is has the wrong sign. Hence the euclidean bosonic two point function (3.9) is not the correlation function of a nonnegative measure. This problem has already been seen by Nicolai for the WZ model in four dimensions [32]. In [19] Jaffe and Lesniewski give an erroneous statement about the positivity of the bosonic measure. We shall see in the next section that (3.9) nevertheless satisfies Osterwalder-Schrader positivity. The antisymmetric kernel $\mu(x, y)=-\mu^{\mathrm{T}}(y, x)$ in (3.8) is that of the Majorana fermion (3.7) for all admitted dimensions. This kernel is exactly the inverse of the differential operator $C^{T}(m-\not)$ of the free fermionic lagrangean in (1.2).

\subsection{Osterwalder-Schrader Positivity}

The link between the Schwinger functions and the physical Hilbert space is Osterwalder-Schrader positivity. Following [34] and [10] we first define a linear operator $\theta$ on the test function space $S$ which reflects the cartesian coordinate $x_{\mathrm{d}}$ of $x=\left(\vec{x}, x_{d}\right) \in R^{d}$. For a column vector $f \in S(\Xi)=S\left(R^{d}\right) \bullet C^{N}$ it has the form

$$
(\theta f)(x)=\mu f\left(\vec{x},-x_{d}\right)
$$

with a matrix $\gamma$ which depends on the spin and satisfies $p^{2}=$ id. For the one component test function of a neutral (pseudo) scalar field it is simply $(\theta f)(x)=$ $f\left(\vec{x},-x_{d}\right)$ and for Dirac spinors with $f \in S\left(\mathbb{R}^{d}\right) \bullet c^{2 N}$ it takes the form $(\theta f)(x)=$ $\left[\begin{array}{ll}\gamma_{d} & 0 \\ 0 & \bar{\gamma}_{d}\end{array}\right] f\left(\vec{x}_{,}-x_{d}\right)$ with the hermitean Dirac matrix $\gamma_{d}$ of the coordinate $x_{d}$. The choice (3.7) of the skew symmetric form yields for the Majorana spinors the reflection operator, see Appendix $B,(\theta f)(x)=\gamma_{d} C f\left(\vec{x},-x_{d}\right)$. 
The reflection operator can then be extended to the algebra $\mathcal{A}(\mathcal{S})$ by $\Gamma(\theta)$. The antilinear OS operator is defined as

$$
\mathrm{Tf}=(\theta \mathrm{f})^{*} \quad \text { if } \mathrm{f} \in \mathcal{S} \text { and } \quad \hat{\mathrm{T} F}=(\Gamma(\theta) \mathrm{F})^{*} \text { if } \mathrm{F} \in \mathcal{\Lambda}(\mathcal{S})
$$

It satisfies $\mathrm{T}^{2}=\mathrm{id}$. Then

$$
B(F, G):=\langle S \mid(\hat{T} F) \circ G\rangle
$$

is a sesquilinear but in general not a positive form on $\mathcal{l}(\mathcal{S})$. The crucial step of the Osterwalder-Schrader reconstruction is to restrict the test functions to $S^{>}(\Xi)=$ $\mathcal{S}\left(\mathfrak{A}_{\times} \times \mathbb{R}_{+}^{\mathrm{d}}\right)$, the subset of all functions in $S\left(\mathfrak{A}_{\times} \mathbb{R}^{\mathrm{d}}\right)$ with a support in the closed set $\mathfrak{A}_{\times} \overline{\mathbb{R}_{+}^{\mathrm{d}}}$ where $R_{+}^{d}$ is the open half space $\left\{\left(x_{1}, \ldots, x_{d}\right) \mid x_{d}>0\right\}$. Here we refer to the extended OS positivity, see [36] and [8]. The corresponding algebra $\mathcal{A}\left(\mathcal{S}^{>}\right)=\underset{n=0}{\infty} \ell_{n}\left(\mathcal{S}^{>}\right)$is spanned by functions $F_{n}\left(\alpha^{1}, x^{1}, \ldots, \alpha^{n}, x^{n}\right) \in d_{n}(\mathcal{S})$ which vanish if $x_{d}^{i} \leq 0$ for some $i \in\{1, \ldots, n\}$. A generating functional (3.3) of Schwinger functions is OS positive if $\mathrm{B}(\mathrm{F}, \mathrm{F}) \geq 0$ holds for all $\mathrm{F} \in \mathcal{M}\left(\mathcal{S}^{>}\right)$. Let $\gamma$ be that subset of $\mu\left(S^{>}\right)$for which $\mathrm{B}(\mathrm{F}, \mathrm{F})=0$. Then $\boldsymbol{\gamma}$ is a linear space and $\mathrm{B}(\mathrm{F}, \mathrm{G})$ defines an inner product on $\mu\left(\mathcal{S}^{>}\right) / \boldsymbol{\gamma}$. Osterwalder and Schrader have shown that under the additional assumptions of euclidean invariance, which we shall discuss in the next section, and of the cluster property the closure of $A\left(S^{>}\right) / \mathcal{H}$ is isomorphic to the physical Hilbert space of the minkowskian quantum field theory. The physical one-particle space is isomorphic to the closure of $\mathcal{S}^{>} / \mathcal{S}_{0}$ with $\mathcal{S}_{0}=\left\{\mathrm{f} \mid \omega(\mathrm{Tf}, \mathrm{f})=0, \mathrm{f} \in \mathcal{S}^{>}\right\}=\gamma \cap \mathcal{S}$.

The generating functional of a free field theory is the exponential (2.11) of the bilinear form $\omega(f, g)=\langle\Omega \mid f \circ g\rangle$ of the two point functions. In this case the OS positivity of (3.12) follows already from the OS positivity of the two point functions

$$
w(\mathrm{Tf}, \mathrm{f}) \geq 0 \quad \text { if } f \in \mathcal{S}^{>} .
$$


This statement is well known, but nevertheless we indicate the proof. The bilinear form $\omega$ is hermitean, i.e. $\omega(\mathrm{Tf}, \mathrm{g})=\bar{\omega}\left(\mathrm{T}_{\mathrm{g}, \mathrm{f})}\right.$ for $\mathrm{f}, \mathrm{g} \in \mathcal{S}$. If $\mathrm{f}, \mathrm{g} \in \mathcal{S}^{>}$that is an immediate consequence of (3.13); for arbitrary functions $f, g \in \mathcal{S}$ it follows with the help of euclidean invariance or by the explicit calculations of Appendix B. Hence we know that $\hat{\mathrm{T}} \Omega=\Omega$, and $\langle\Omega \mid \mathrm{Tf} \circ \mathrm{g}\rangle$ can be written as $(\mathrm{f} \mid \mathrm{Mg})$ with the inner product (2.1) of $\boldsymbol{x}$ and a hermitean operator $M$ for all $f, g \in \mathcal{S}$. This operator can be extended to the algebra $\mathcal{A}\left(\mathcal{S}^{>}\right)$by $\Gamma(\mathrm{M})$. From (3.13) we obtain (f|Mf) $\geq 0$ if $\mathrm{f} \in \mathcal{S}^{>}$, and consequently $(F \mid \Gamma(M) F) \geq 0$ if $F \in \mathcal{A}\left(\mathcal{S}^{>}\right)$. As generalization of $\langle\Omega \mid T f \circ g\rangle=(f \mid M g)$ the sesquilinear form (3.12) can be identified with

$$
<\exp \Omega|(\hat{\mathrm{T} F}) \circ G>=(\exp \Omega\lrcorner \mathbf{F}| \Gamma(\mathbf{M})(\exp \Omega\lrcorner G))
$$

This identity follows from (2.11), see eqs. (3.9) and (3.10) in [23]. The interior product (2.13) with $\exp \Omega$ takes into account all possible contractions with the tensor $\Omega$. These contractions do not change the support: if $F \in \mathcal{A}\left(\mathcal{S}^{>}\right)$then $\exp \Omega\lrcorner F \in \mathcal{H}\left(S^{>}\right)$. Since $\Gamma(M)$ is positive on $\mathcal{A}\left(\mathcal{S}^{>}\right)$, the identity (3.14) yields the positivity of $\langle\exp \Omega| \hat{\mathrm{T} F} \circ \mathrm{F}>$ if $F \in \mathcal{A}\left(\mathcal{S}^{>}\right)$.

The WZ theory in two dimensions which we investigate in some detail has the bilinear form (3.8) with test functions $f, g \in S\left(\mathbb{R}^{2}\right) \bullet C^{4}$. The kernels $D$ and $\boldsymbol{h}$ have already been specified in (3.9) and (3.7). The OS operator for this combined theory is given by

$$
T f(x)=\left[\begin{array}{cc}
1_{2} & 0 \\
0 & \bar{\gamma}_{2} C
\end{array}\right] f\left(x_{1},-x_{2}\right)
$$

with the $2 \times 2$ unit matrix $1_{2}$. The results of Appendix B imply that $w(T f, f) \geq 0$ if $f \in \mathcal{S}\left(\mathbb{R}_{+}^{2}\right) \bullet C^{4}=\mathcal{S}^{>}$. Due to the restriction of the support of the test functions the $\delta$-function term in (3.9) does not contribute to the OS-form. 


\subsection{Euclidean Invariance and Lorentz Invariance}

In this Section we recapitulate well known facts about the relation between euclidean invariance of the functionals and Lorentz invariance of the physical theory, see $[34,12]$. This is the basis of a straightforward extension to euclidean supersymmetry presented in the next section.

Let $S(\Xi)=S\left(R^{\mathrm{d}}\right) \bullet \mathrm{C}^{\mathrm{N}}$ be the test function space of a theory with several particles with spin as considered in Sect. 2.1. Then $\mathcal{S}(\Xi)$ carries a representation of the euclidean group $S O(d) \times R^{d}$, the semidirect product of the rotation group $S O(d)$ and the translation group $\mathbb{R}^{\mathrm{d}}$, which corresponds to the behaviour of classical fields. More precisely there is a faithful represention of the twofold covering group $\tilde{S} O(d) \times R^{d}$ on the test function space $U(\tilde{R}, a) f(\alpha, x)=\sum_{\beta} D_{\alpha \beta}(\tilde{R}) f\left(\beta, R^{-1}(x-a)\right)$ for $\tilde{R} \in \tilde{S} O(d)$ and $a \in \mathbf{R}^{d}$, with a unitary finite dimensional representation $\mathcal{D}(\tilde{\mathrm{R}})$ of $\tilde{\mathrm{S}} \mathrm{O}(\mathrm{d})$ which decomposes into irreducible parts according to the spins of the particles involved. For $d \geq 3$ the twofold covering group of $\mathrm{SO}(\mathrm{d})$ is also the universal covering group. Quantum fields with intermediate statistics for $d=2$ or 3 are excluded by our choice of the test function algebra. Details of these representations in $d=4$ dimensions can be found in [36]. This representation $U$ on $\mathcal{S}(\Xi)$ is then extended to a representation $\Gamma(U)$ on the algebra $\mathcal{A}(\mathcal{S})$. Euclidean invariance of the quantum field theory is the statement that $\langle\mathrm{S} \mid \Gamma(\mathrm{U}(\tilde{\mathrm{R}}, \mathrm{a})) \mathrm{F}\rangle=\langle\mathrm{S} \mid \mathrm{F}\rangle$ for all $\tilde{\mathbf{R}} \in \tilde{\mathrm{S}} \mathrm{O}(\mathrm{d}), a \in \mathbb{R}^{\mathrm{d}}$ and for all test functions $\mathrm{F} \in \mathcal{\mu}(\mathcal{S})$.

To extend euclidean invariance to euclidean supersymmetry it is more convenient to use infinitesimal transformations. The real Lie algebra $\mathcal{E}_{0}^{\mathbb{R}}$ of the representation of the euclidean group given above is generated by the differential operators

$$
\mathbf{M}_{\mu \nu}=-\left(\mathbf{x}_{\mu} \partial_{\nu}-\mathbf{x}_{\nu} \partial_{\mu}\right)+S_{\mu \nu}, \mathbf{M}_{\mu}=-\partial_{\mu}
$$


with $\mu, \nu=1, \ldots, \mathrm{d}$. Here $\mathrm{S}_{\mu \nu}$ is a finite dimensional antihermitean spin representation. In the case of Dirac or Majorana spinors it reads $S_{\mu \nu}=\sigma_{\mu \nu}^{\mathrm{T}}$ with $\sigma_{\mu \nu}$ $=\frac{1}{4}\left(\gamma_{\mu} \gamma_{\nu}-\gamma_{\nu} \gamma_{\mu}\right)$. The transposed operator $\sigma_{\mu \nu}^{\mathrm{T}}$ of the spin generator originates from our conventions of duality between test functions and functionals. The skew symmetric Lie product of the algebra $\mathcal{E}_{0}^{R}$ is the commutator. All operators of $\mathcal{E}_{0}^{\mathbb{R}}$ are antihermitean with respect to the inner product (2.1), they are continuous in the nuclear topology of $\mathcal{S}$. The Lie algebra of the representation $\Gamma(\mathrm{U}(\tilde{\mathrm{R}}, \mathrm{a}))$ on $\lambda_{(\gamma)}(\mathcal{S})$ is exactly the real linear space of all derivations $D_{M}, M \in \varepsilon_{0}^{R}$. The operators $D_{M}$ are continuous mappings from $\lambda_{(\gamma)}(\mathcal{S})$ into $\lambda_{(\gamma-1)}(\mathcal{S})$. The $\mathrm{C}$-linear span of $\mathcal{E}_{0}^{\mathrm{R}}$ will be denoted by $\varepsilon_{0}^{C}$.

The euclidean invariance $\langle\mathrm{S} \mid \Gamma(\mathrm{U}(\tilde{\mathrm{R}}, \mathrm{a})) \mathrm{F}\rangle=\langle\mathrm{S} \mid \mathrm{F}\rangle$ is equivalent to

$$
<S \mid D_{M} F>=0
$$

for any $M \in \mathcal{E}_{0}^{\mathbb{R}}$ and all test functions $F \in \mathcal{A}(\mathcal{S})$. But since $D_{\lambda M}=\lambda D_{M}$ for $\lambda \in C$ this identity holds for any $M \in \varepsilon_{0}^{C}$. The generators (3.16) satisfy the following commutation relations with the antilinear OS operator

$$
\mathrm{TM}=\epsilon \mathbf{M T}
$$

with $\epsilon=+1$ if $\mathbf{M}=\mathbf{M}_{\mu}$ or $\mathbf{M}_{\mu \nu}$ with $\mu, \nu=1, \ldots, \mathrm{d}-1$ and with $\epsilon=-1$ if $\mathbf{M}=\mathbf{M}_{\mathrm{d}}$ or $M_{\mu \mathrm{d}}$. The derivations $D_{M}$ then satisfy the relation

$$
\hat{\mathrm{T}} \mathrm{D}_{\mathbf{M}}=\mathrm{D}_{\epsilon \mathbf{M}} \hat{\mathrm{T}}
$$

From the Leibniz rule we know $D_{M}(\hat{T} F \circ G)=\left(D_{M} \hat{T F}\right) \circ G+\hat{T F} D_{M} G=$ $\left(\hat{\mathrm{T} D} \mathrm{CM}_{\mathrm{M}} \mathrm{F}\right) \circ \mathrm{G}+\hat{\mathrm{T} F}_{\mathrm{M}} \mathrm{D}_{\mathbf{M}} \mathrm{G}$ for all $\mathrm{F}, \mathrm{G} \in \mathcal{\Lambda}(\mathcal{S})$. Euclidean invariance (3.17) then implies 


$$
<S \mid D_{M}(\hat{T F \circ G})>=B\left(D_{\epsilon M} F, G\right)+B\left(F, D_{M} G\right)=0
$$

with the OS form (3.12). If we restrict this identity to the space $S$ is reads

$$
\epsilon \omega(\mathrm{TMf}, \mathrm{g})+\omega(\mathrm{Tf}, \mathrm{Mg})=0
$$

with $f, g \in S$ and $\epsilon= \pm 1$ specified as in (3.18). The operators $M \in \mathcal{E}_{0}$ are differential operators on $S$ which map $\mathcal{S}^{>}$into itself. The relation (3.21) implies that the operators

$$
\mathrm{J}_{\mu \nu}:=-\mathrm{i} \mathbf{M}_{\mu \nu^{\prime}} \quad \mathrm{J}_{\mu 0}:=\mathbf{M}_{\mu \mathrm{d}}, \quad \mathrm{P}_{\mu}:=-\mathrm{i} \mathbf{M}_{\mu^{\prime}} \quad \mathrm{P}_{0}:=\mathbf{M}_{\mathrm{d}}
$$

with $\mu, \nu=1, \ldots, \mathrm{d}-1$ are hermitean operators on $\mathcal{S}^{>}$with respect to the non negative OS form $\omega(\mathrm{Tf}, \mathrm{g})$. A more detailed investigation, see $[34,12]$, shows that these operators map the null space $\mathcal{S}_{0}$ into itself and have a total set of analytic vectors in $s^{>} / \mathcal{S}_{0}$. Hence they are essentially selfadjoint operators on $\mathcal{S}^{>} / \mathcal{S}_{0}$. It is straightforward to verify that the $\mathbb{R}$-linear span of the operators $\mathrm{J}_{\mu \nu}$ and $\mathrm{P}_{\mu}$ with $\mu, \nu=0,1, \ldots, \mathrm{d}-1$ forms the real Lie algebra $\mathbb{L}_{0}^{\mathbb{R}}$ of the inhomogeneous Lorentz group, the Lie product given by $\mathrm{i}[\cdot, \cdot]$. The same argument can be applied to the derivations $\mathrm{D}_{\mathbf{M}}$ on $\mathcal{A}(\mathcal{S})$, the hermiticity of the corresponding derivations follows immediately from (3.20). The complex Lie algebra $\left\{D_{M} \mid M \in \mathcal{E}_{0}^{\mathbb{C}}\right\}$ includes the real Lie algebra $\left\{D_{M} \mid M \in \mathcal{L}_{0}^{\mathbb{R}}\right\}$ of the inhomogeneous Lorentz group, the elements of which are exactly the hermitean operators with respect to the OS form (3.12). The physical theory constructed with (3.12) on the closure $\overline{A\left(\mathcal{S}^{>}\right) / \delta}$ has therefore the correct relativistic covariance. 


\subsection{Supersymmetry}

The generators of the euclidean group (3.16) are even operators on the test function space $\mathcal{S}$. This complex Lie algebra $\mathcal{E}_{0}$ can be extended to a complex Lie superalgebra of differential operators with the following properties

i) The even part of $\mathcal{E}^{\mathbf{C}}$ is $\mathcal{E}_{0}^{\mathbf{C}}$.

ii) The odd part $\varepsilon_{1}^{C}$ is given by the linear span of spinorial type operators $Q_{\alpha}$, $\alpha=1, \ldots, N_{d}$, i.e. $Q(\eta)=\sum_{\alpha} Q_{\alpha} \eta_{\alpha}$ with a numerical spinor $\eta \in C^{N_{d}}$.

The algebraic relations between the generators are

$$
\begin{aligned}
& {\left[\mathrm{M}_{\mu}, \mathrm{Q}(\eta)\right]=0,} \\
& {\left[\mathrm{M}_{\mu \nu}, \mathrm{Q}(\eta)\right]=-\mathrm{Q}\left(\sigma_{\mu \nu} \eta\right), \quad \mu, \nu=1, \ldots, \mathrm{d} .} \\
& \left\{\mathrm{Q}(\eta), \mathrm{Q}\left(\eta^{\prime}\right)\right\}=-2 \underset{\mu}{\Sigma}\left(\eta^{\mathrm{T}} \gamma_{\mu^{\prime}} \eta^{\prime}\right) \mathrm{M}_{\mu^{\prime}}
\end{aligned}
$$

iii) The commutation relations with the OS operator $\mathrm{T}$ are

$$
\mathrm{TQ}(\eta) \mathrm{Tf}=(-1)^{\pi(\tilde{f})} \mathrm{Q}(\tilde{\eta}) \mathrm{f} \quad \text { with } \tilde{\eta}=\mathrm{C} \overline{\gamma_{\mathrm{d}}} \bar{\eta}
$$

Explicit examples for these operators will be given in Sect. 4.1. All elements of $\mathcal{E}$ are differential operators on $\mathcal{S}$, continuous in the nuclear topology. They determine by (2.9) a class of superderivations $\left\{D_{L} \mid L \in \mathcal{E}^{\mathbf{C}}\right\}$ operating on the test function algebra $\mu(\mathcal{S})$.

A quantum field theory with generating functional (3.3) is supersymmetric if

$$
<\mathrm{S}\left|\mathrm{D}_{\mathrm{L}} \mathrm{F}\right\rangle=0
$$


holds for all $\mathrm{L} \in \mathcal{E}^{C}$ and any $F \in \mathcal{A}(S)$. This identity is the essential dynamical statement; the mere existence of the Lie superalgebra $\mathcal{E}^{\mathbf{C}}$ does not yet imply supersymmetry. Before we relate this definition of supersymmetry with the usual definition for a minkowskian quantum field theory we give some comments to the conditions i) - iii). The algebraic relations between the generators (3.16) of the euclidean group and the relations (3.23) including the "supercharge" operators $Q(\eta)$ constitute the complex euclidean Lie superalgebra $\mathcal{E}^{\mathbb{C}}$. This algebra has been investigated in the literature, see e.g. $[27,28]$. But the search for a real euclidean superalgebra leads to inconsistencies [29]. In fact these investigations show that there is no reasonable reality/hermiticity condition in a purely euclidean setting. The only conjugation which allows a meaningful construction of a real algebra is the OS involution (3.11). If we start from an ansatz $T Q(\eta) T=Q(\Lambda \bar{\eta}) J$ with a matrix $\Lambda$ and a linear operator $\mathrm{J}$ which satisfies $\mathrm{J}^{2}=\mathrm{id}$, then the relations (3.18) together with (3.23) can be satisfied only if $J$ is the parity operator $J f=(-1)^{\pi(f)_{f}}$ and the matrix $\Lambda$ has to be $\Lambda=C \overline{\gamma_{d}}$. Hence (3.25) is essentially a consequence of (3.18) and (3.23).

To derive supersymmetry for the reconstructed physical theory we first extend (3.24) to the operators $\mathrm{D}_{\mathrm{Q}}$ and $\hat{\mathrm{T}}$ on $\mathcal{A}(\mathcal{S})$. A simple calculation yields

$$
\mathrm{D}_{\mathrm{Q}} \hat{\mathrm{TF}}=(-1)^{\pi(\mathrm{F})} \hat{\mathrm{T}} \mathrm{D}_{Q^{F}}
$$

with $Q=Q(\eta)$ and $Q=Q(\tilde{\eta})$ for any $F \in \mathcal{A}(S)$. We can now follow the arguments of Sect. 3.3 using the supersymmetry statement (3.25) to prove that $D_{Q(\eta)}$ is a hermitean operator with respect to the OS form (3.12) on $A\left(\mathcal{S}^{>}\right)$if $\eta$ satisfies the Majorana condition of a minkowskian spinor field

$$
\eta=-\mathrm{C} \overline{\gamma_{\mathrm{d}}} \bar{\eta}=-\mathrm{C} \boldsymbol{\gamma}_{\mathrm{d}}^{\mathrm{T}} \bar{\eta}
$$


The identity (3.26) takes over the rôle of (3.19) and the parity factor $(-1)^{\pi(F)}$ is exactly compensated by the parity factor of the modified Leibniz rule for the antiderivation $\mathrm{D}_{\mathrm{Q}}$. The restriction to $\mathcal{S}^{>}$yields that $\mathrm{Q}(\eta)$ is hermitean with respect to $\omega(\mathrm{Tf}, \mathrm{g})$ if $\eta$ satisfies (3.27). From the last equation of (3.23) we obtain

$$
(\mathrm{Q}(\eta))^{2}=\sum_{\mu=1}^{\mathrm{d}}\left(\eta^{+} \gamma_{\mathrm{d}} \gamma_{\mu} \eta\right) \mathrm{M}_{\mu}=\left(\eta^{+} \eta\right) \mathrm{P}_{0}-\sum_{\mathbf{k}=1}^{\mathrm{d}-1} \mathrm{i}\left(\eta^{+} \gamma_{\mathrm{d}} \gamma_{\mathbf{k}} \eta\right) \mathrm{P}_{\mathbf{k}}
$$

where $i \eta^{+} \gamma_{\mathrm{d}} \gamma_{\mathbf{k}} \eta, \mathbf{k}=1, \ldots, \mathrm{d}-1$, are real coefficients. Hence with $\mathrm{P}_{\mu}$ also the operators $\mathrm{Q}(\eta)$ have a total set of analytic vectors in $\mathcal{S}^{>} / \mathcal{S}_{0}$ and are essentially selfadjoint on that domain. In the same way we obtain that the antiderivations $\mathrm{D}_{\mathrm{Q}(\eta)}$ with $\eta$ restricted by (3.27) are essentially selfadjoint on $\mathcal{A}\left(\mathcal{S}^{>}\right) / \ell$. Moreover eq. (3.28) implies that the hamiltonian of the theory $\mathrm{D}_{\mathrm{P}_{0}}$ can be represented as the square of a "supercharge" $\mathrm{D}_{\mathrm{Q}}$.

The main conclusion of this section can be formulated as follows: For a supersymmetric quantum field theory there exists a complex euclidean Lie superalgebra $\varepsilon^{C}$ of continuous differential operators on the test function space $\mathcal{S}$. These operators induce a representation $D_{L}$ of superderivations which are infinitesimal symmetry operators in the sense of (3.25). The OS form (3.12) B(F,G) selects a subspace of hermitean operators $\left\{D_{L} \mid L \in \mathcal{L}^{\mathbb{R}}\right\}$ where $\mathscr{L}^{\mathbb{R}}$ is the real Lie superalgebra of a minkowskian supersymmetric quantum field theory. The even part $\mathcal{L}_{0}^{R}$ is the Lie algebra of the inhomogeneous Lorentz group considered in Section 3.3, and the odd part $\digamma_{1}$ is spanned by the Majorana type supercharges $Q(\eta)$ with $\eta$ restricted by (3.27). 


\section{The Wess-Zumino Model}

To exemplify our definition of supersymmetry we shall use the WZ model in two dimensions. The consistency with the usual formulations can already be seen at the free WZ model. For the interacting model we shall present formal agruments which are rigorous only on the level of perturbation theory with UV regularization, but nevertheless they show that the mathematics presented here is an adequate framework for the construction of supersymmetric models. Finally we indicate how these formal arguments can be transferred to a rigorous construction related to the work of Jaffe, Lesniewski and Weitsman [17] and Arai [1]. Up to this last step all calculations can be done also for $d=3$ or 4 dimensions. The restriction to $d=2$ is only used to get simpler expressions.

\subsection{The Free WZ Model}

The Schwinger functions of a free quantum field theory are generated by the exponential (2.11) of the bilinear form (3.4) $\omega(f, g)=\langle\Omega \mid f \circ g\rangle$ for the two point functions. Let us assume that $\Omega \in \Lambda_{2}\left(x_{(-p)}\right)$ for some $p>0$, then $\exp \Omega$ converges within the Fock space $\mathcal{F}_{(-\gamma)}\left(\boldsymbol{x}_{(-\mathrm{p})}\right)$ and consequently in the algebra $\mathcal{A}_{(-\gamma)}\left(\mathcal{S}^{\prime}\right)$ if $\gamma<0$. The supersymmetry statement $(3.28)$ is equivalent to $\langle\exp \Omega| \mathrm{D}_{\mathrm{L}} \mathrm{H}>$ $=\left\langle D_{L^{\prime}}\right.$ exp $\left.\Omega\right| H>=0$ for all $H \in \mathcal{A}(\mathcal{S}) \subset \Lambda_{(\gamma)}(\mathcal{S})$ and $L \in \mathcal{E}^{C}$ with the dual operator $L^{\prime}$ of $L$, see (2.12). Since $D_{L^{\prime}}$ is a superderivation and $\Omega$ has always even parity, we have $D_{L}, \exp \Omega=\left(D_{L}, \Omega\right) \circ \exp \Omega$. Supersymmetry of a free field theory is therefore equivalent to the supersymmetry $D_{L^{\prime}}, \Omega=0$ of the two point functions, a fact which is of course well known from the minkowskian approach. The identity $D_{L}, \Omega=0$ is now evaluated as

$$
<\Omega\left|D_{M}(f \circ g)>=<\Omega\right| M f \circ g>+<\Omega \mid f \circ M g>=\omega(M f, g)+\omega(f, M g)=0
$$

for all $f, g \in S$ if $M \in \mathcal{E}_{0}^{C}$ is a generator of a euclidean transformation, and as 


$$
\begin{gathered}
<\Omega \mid D_{Q}(f \circ g)>=\left\langle\Omega\left|Q f \circ g>+(-1)^{\pi(f)}<\Omega\right| f \circ Q g>\right. \\
=\omega(Q f, g)+(-1)^{\pi(f)} \omega(f, Q g)=0
\end{gathered}
$$

if $Q \in \mathcal{E}_{1}$ is a proper supersymmetry transformation.

For the detailed calculation we take the neutral $\mathrm{WZ}$ model in $\mathrm{d}=\mathbf{2}$ dimensions as example. The test function space $S=S\left(\mathbb{R}^{2}\right) \bullet \mathrm{C}^{4}$ is embedded into the Hilbert space $\boldsymbol{x}=\mathcal{L}^{2}\left(\mathbb{R}^{2}\right) \bullet \mathcal{C}^{4}$. In an explicit representation we shall write $\boldsymbol{x}=\boldsymbol{x}_{\mathrm{B}} \bullet \boldsymbol{x}_{\mathrm{F}}$ $=x_{\mathrm{a}} \bullet x_{\mathrm{b}} \bullet x_{\mathrm{F}}$, i.e. $\mathrm{f}(\mathrm{x})=\left[\begin{array}{l}\mathrm{a}(\mathrm{x}) \\ \mathrm{b} \\ \chi(\mathrm{x})\end{array}\right] \in x$ with $\mathrm{a}(\mathrm{x}) \in x_{\mathrm{a}}=\mathcal{L}^{2}\left(\mathbb{R}^{2}\right), \mathrm{b}(\mathrm{x}) \in x_{\mathrm{b}}=\mathcal{L}^{2}\left(\mathbb{R}^{2}\right)$, $x_{\mathrm{B}}=\boldsymbol{x}_{\mathrm{a}} \bullet \boldsymbol{x}_{\mathrm{b}}$ being the bosonic subspace, and the spinor function $\chi(x) \in \boldsymbol{x}_{\mathrm{F}}$ $=\mathcal{L}^{2}\left(\mathbb{R}^{2}\right) \bullet C^{2}$. The fundamental bilinear form on $x$ is

$$
\left\langle f_{1}\right| f_{2}>=\int\left(a_{1}(x) a_{2}(x)+b_{1}(x) b_{2}(x)+\sum_{\alpha=1}^{2} \chi_{(1) \alpha}(x) \chi_{(2) \alpha}(x)\right) d x
$$

The test function space is split accordingly into $S=\mathcal{S}_{\mathrm{B}} \bullet \mathcal{S}_{\mathrm{F}}=\mathcal{S}_{\mathrm{a}} \bullet \mathcal{S}_{\mathrm{b}} \bullet \mathcal{S}_{\mathrm{F}}$. The bilinear form for the two point functions has already been given in (3.8). The bosonic kernel (3.9) and the fermionic kernel (3.7) are obtained by inversion of the differential operators which show up in the free lagrangean (1.2). As already known from Sect. 3 the solutions for (4.1) are the generators (3.16) of the euclidean transformations. The explicit form of the generator of the rotations is $M_{12} f(x)=-\left(x_{1} \partial_{2}-x_{2} \partial_{1}\right) f(x)+\left[\begin{array}{ll}0 & 0 \\ 0 & \sigma^{x}\end{array}\right] f(x)$ with $\sigma=\gamma_{1} \gamma_{2}$. The solutions for (4.2) are odd operators $Q$ which satisfy

$$
\mathbf{Q}^{\mathbf{T}} \mathbf{K}+\mathrm{JKQ}=0
$$

where $K$ is the kernel of $\omega(f, g)$, see $(3.8)$, J is the parity operator $J f=(-1)^{\pi(f)_{f} \text { and }}$ $\mathrm{Q}^{\mathrm{T}}$ is the transposed operator (with respect to the form (4.3)). This equation is solved 
by the ansatz $Q=\left[\begin{array}{cc}0 & R_{1} \\ R_{2} & 0\end{array}\right]$ if the operators $R_{1}: \mathcal{S}_{F} \rightarrow \mathcal{S}_{B}$ and $R_{2}: \mathcal{S}_{\mathrm{B}} \rightarrow \mathcal{S}_{\mathrm{F}}$ satisfy the constraint $R_{1}^{T} D-\mu R_{2}=0$. The solutions can be labeled by a spinor $\eta \in C^{2}$ and yield the final representation

$$
\mathrm{Q}(\eta) \mathrm{f}(\mathrm{x})=\left[\begin{array}{l}
\eta^{\mathrm{T}} \boldsymbol{g}^{\mathrm{T}} \chi(\mathrm{x}) \\
\eta^{\mathrm{T}} \chi(\mathrm{x}) \\
\mathrm{a}(\mathrm{x}) \mathrm{C} \eta+\mathrm{C} \not \partial \mathrm{b}(\mathrm{x}) \eta
\end{array}\right]
$$

These supersymmetry transformations operate on test functions. The dual (transposed) transformations operate on Schwinger functions, i.e. formally on euclidean fields which live in the dual space $\mathcal{S}^{\prime}$. The duality pairing between $f \in \mathcal{S}$ and $\Phi=\left[\begin{array}{l}\mathrm{A} \\ \mathrm{B} \\ \Psi\end{array}\right] \in \mathcal{S}^{\prime}$ is given by (4.3). The dual supersymmetry transformation (which on $\mathcal{C}^{2}\left(\mathbb{R}^{2}\right) \bullet C^{4}$ coincides with the transposed operator $Q^{T}$ )

$$
\mathrm{Q}^{\prime}(\eta) \Phi(\mathrm{x})=\left[\begin{array}{l}
\eta_{\mathrm{C}}^{\mathrm{T}} \Psi(\mathrm{x}) \\
\eta^{\mathrm{T}} \mathrm{C}^{\mathrm{T}} \boldsymbol{\Psi}(\mathrm{x}) \\
\partial \mathrm{A}(\mathrm{x}) \eta+\mathrm{B}(\mathrm{x}) \eta
\end{array}\right]
$$

is the euclidean version of the field transformations of the WZ model in two dimensions, see [47]. To derive the operators (4.5) and (4.6) from the symmetry condition (4.2) it is essential that the bosonic part (3.9) of the bilinear form (3.8) includes the $\delta$-function. For the free WZ model such a term, which does not contribute to the OS form on $\mathcal{A}\left(\mathcal{S}^{>}\right)$, can be eliminated and there are still modified supersymmetry transformations $Q$ (as is well known for the minkowskian model). But the extended version derived from the lagrangean (1.2) will be needed for the model with interaction. 


\subsection{Perturbation Theory}

The interaction lagrangean of a local field theory is a polynomial in the interacting fields with a kernel $\Gamma \in \mathcal{\Lambda}\left(\mathcal{S}^{\prime}\right)$ which has even parity. In the case of the WZ model the interaction part (1.3) is a polynomial of third degree which corresponds to the following 3-linear form in the field vectors $\Phi_{i}=\left[\begin{array}{c}A_{i} \\ B_{i}^{i} \\ \Psi_{i}^{i}\end{array}\right] \in \mathcal{S}^{\prime}=S^{\prime}\left(R^{2}\right) \bullet C^{4}, i=1,2,3$,

$$
\begin{aligned}
& <\Phi_{1} \circ \Phi_{2} \circ \Phi_{3}|\Gamma\rangle= \\
& \int \sigma\left(x^{1}, x^{2}, x^{3}\right)\left[A_{1}\left(x^{1}\right) A_{2}\left(x^{2}\right) B_{3}\left(x^{3}\right)+A_{2}\left(x^{1}\right) A_{3}\left(x^{2}\right) B_{1}\left(x^{3}\right)\right. \\
& \left.+A_{3}\left(x^{1}\right) A\left(x^{2}\right) B\left(x^{3}\right)\right] d x^{1} d x^{2} d x^{3} \\
& -\int \sigma\left(x^{1}, x^{2}, x^{3}\right)\left[A_{1}\left(x^{1}\right) \Psi_{2}^{T}\left(x^{2}\right) C \Psi_{3}\left(x^{3}\right)-A_{2}\left(x^{1}\right) \Psi_{3}^{T}\left(x^{2}\right) C \Psi_{1}\left(x^{3}\right)\right. \\
& \left.+A_{3}\left(x^{1}\right) \Psi_{1}^{T}\left(x^{2}\right) C \Psi_{2}\left(x^{3}\right)\right] d x^{1} d x^{2} d x^{3}
\end{aligned}
$$

Here $\sigma\left(x^{1}, x^{2}, x^{2}\right)=\delta\left(x^{1}-x^{2}\right) \delta\left(x^{2}-x^{3}\right)$ is the local coupling of the unregularized theory. With this kernel the expression (4.7) is defined only for a restricted class of fields $\Phi$. For the further investigations we introduce an UV regularization

$$
\sigma\left(x^{1}, x^{2}, x^{3}\right)=\int \kappa\left(x^{1}-y\right) \kappa\left(x^{2}-y\right) \kappa\left(x^{3}-y\right) d y
$$

with a smooth function $\kappa(x) \in \mathcal{S}\left(\mathbb{R}^{2}\right)$ with $\kappa(x) \geq 0, \quad \operatorname{supp} \kappa c\{x|| x \mid \leq 1\}$ and $\int k(x) d x=1$. This ansatz may be obtained by smearing all fields with the same function $k(x)$ as done by Nicolai in [32] for the WZ model in four dimensions. The function (4.8) is totally symmetric and translation and rotation invariant. The regularization is removed by the substitution $\kappa(x) \rightarrow \kappa_{\lambda}(x)=\lambda^{-1} \kappa\left(\lambda^{-1} x\right), 0<\lambda \leq 1$, in the limit $\lambda \rightarrow 0$. The (regularized) interaction lagrangean is supersymmetric, i.e. 


$$
D_{L} \Gamma=0
$$

for all $L \in \mathcal{E}^{\mathbf{C}}$. This identity is equivalent to the usual (super)symmetry statements $0=\delta\left\langle\Phi_{1} \circ \Phi_{2} \circ \Phi_{3} \mid \Gamma\right\rangle:=\left\langle D_{L^{\prime}}\left(\Phi_{1} \circ \Phi_{2} \circ \Phi_{3}\right) \mid \Gamma\right\rangle=\left\langle\Phi_{1} \circ \Phi_{2} \circ \Phi_{3} \mid D_{L} \Gamma\right\rangle$ where the $\Phi$ 's are submitted to the field transformations (4.6) (or the corresponding euclidean transformations). The last equality follows from (2.12).

To define the generating functional for the interacting theory one needs (at least in intermediate steps) a regularized tensor $\Gamma$ which is an element of the test function algebra. That has not yet been achieved with the translational invariant regularization (4.8); one needs an additional volume cutoff. E.g. (4.8) may be amended by $\vartheta\left(\rho^{-1} x^{3}\right) \sigma\left(x^{1}, x^{2}, x^{3}\right)$ with $\vartheta(x) \in C^{\infty}\left(R^{2}\right), \mathscr{v}(x)=1$ if $|x|<1, \mathscr{v}(x)=0$ if $|x|>2$, where $\rho \in[1, \infty)$ is a cutoff parameter. Another choice would be the restriction to a torus of size $\rho$ with appropriate boundary conditions, see $[16,17]$. We shall denote each of these kernels with UV regularization and volume cutoff by $\sigma_{\rho}\left(x^{1}, x^{2}, x^{3}\right)$. The unpleasant consequence of any volume cutoff is that $\Gamma$ is no longer translation invariant, and complete supersymmetry can be established only after the cutoff has been removed $(\rho \rightarrow \infty)$. The generating functional for the interacting theory is formally given by

$$
<\mathrm{S}|\mathrm{H}\rangle=\frac{\langle\exp \Omega \mid(\exp -\lambda \Gamma) \circ \mathrm{H}\rangle}{\langle\exp \Omega| \exp -\lambda \Gamma>}, \quad \mathrm{H} \in \mathcal{A}(\mathcal{S})
$$

The terms of the power series expansion in the coupling parameter $\lambda$ are defined if $\Gamma \in \mathcal{A}(\mathcal{S})$, i.e. for an interaction with $U V$ regularization and volume cutoff. But this series does not absolutely converge, and the definition of (4.10) beyond perturbation theory is a serious problem even with all regularizations. We shall come back to that problem in the next section. From the terms of the power series expansion one can extract factors $\left\langle\Omega^{m} \mid \Gamma^{n}\right\rangle, 2 m=3 n$, which are not linked to the external test funciions (here $\Gamma^{\mathbf{n}}$ is the $\mathrm{n}$-th power $\Gamma \circ \ldots . . \Gamma$ ) 


$$
<\exp \Omega \mid \Gamma^{\mathrm{n}} \circ \mathrm{H}>=\underset{\mathrm{p}+\mathrm{q}=\mathrm{n}}{\mathrm{p} ! \mathrm{q} !}\left\langle\exp \Omega\left|\Gamma^{\mathrm{p}}><\exp \Omega\right| \Gamma^{\mathrm{q}} \circ \mathrm{H}>^{\ell}\right.
$$

The linked terms $<\exp \Omega \mid \Gamma^{\mathrm{q}_{\mathrm{oH}}}>^{\ell}\left(=\frac{1}{\mathrm{~m} !}<\Omega^{\mathrm{m}}\left|\Gamma^{\mathrm{q}_{\mathrm{oH}}}\right\rangle^{\ell}\right.$ with $2 \mathrm{~m}=3 \mathrm{q}+\mathrm{n}$ if H $\in \mathcal{h}_{\mathrm{n}}(\mathcal{S})$ ) are sums of those contractions which are connected with the test functions. A proof of this statement follows from (2.11); the sign factor in (2.11) does not show up in (4.11) since $\pi(\Gamma)=0$. For an explicit calculation with hafnians and pfaffians see [6]. Hence (4.10) is equivalent to the linked cluster expansion

$$
<\mathrm{S}|\mathrm{H}\rangle=\sum_{\mathrm{n}=0}^{\infty} \frac{(-\lambda)^{\mathrm{n}}}{\mathrm{n} !}<\exp \Omega\left|\Gamma^{\mathrm{n}} \mathrm{oH}\right\rangle^{\ell}
$$

The linked terms of this perturbation expansion can be defined with the supersymmetric UV regularization (4.8) alone, whereas the factors $\left\langle\Omega^{\mathrm{m}} \mid \Gamma^{\mathrm{n}}\right\rangle$ in (4.11) have a volume divergence. Each linked term corresponds to a finite sum of connected Feynman diagrams. This perturbation theory can of course also be formulated in the language of the Symanzik-Schwinger formalism [9] or in the language of the Berezin integration. An extensive presentation of perturbation and renormalization theory for minkowskian supersymmetry can be found in [37].

Let $\mathrm{D}_{\mathrm{L}}$ be a superderivation with $\mathrm{L} \in \mathcal{E}^{\mathbf{C}}$ then we know $\mathrm{D}_{\mathrm{L}}, \Omega=0$ and moreover $<\exp \Omega\left|D_{L}\left(\Gamma_{\rho}^{n} \circ H\right)>=<\exp \Omega\right|\left(D_{L} \Gamma_{\rho}^{n}\right) \circ H>+<\exp \Omega \mid \Gamma_{\rho}^{n_{o D}} D_{L} H>=0$

where $\Gamma_{\rho} \in \lambda_{3}(\mathcal{S})$ is the interaction tensor with the UV regularization and the volume cutoff. This rule of partial integration can be transferred to the linked terms. But for these terms the limit $\rho \rightarrow \infty$ can be performed and all contributions from $D_{L} \Gamma^{n}=$ $\mathrm{n} \Gamma^{\mathrm{n}-1} \circ \mathrm{D}_{\mathrm{L}} \Gamma$ vanish as a consequence of (4.9). Hence we obtain the supersymmetry identity (3.25)

$$
<\exp \Omega \mid \Gamma^{\mathrm{n}} \circ D_{L}>^{\ell}=0, \quad \mathrm{n}=0,1, \ldots
$$


for each term of the perturbation expansion (4.12). The identities (4.13) correspond to the Ward identities of the usual minkowskian perturbation theory, see e.g. [14] or [37].

\subsection{Beyond Perturbation Theory}

In this section we present a few steps towards a rigorous definition of the generating functional (4.10) without further investigation of OS positivity or supersymmetry. The main emphasis is laid to clarify the following two problems within our functional point of view:

i) The elimination of the auxiliary bosonic degrees of freedom, and

ii) the calculation of functionals for Majorana spinors.

Finally the functional is transferred to an integral which essentially agrees with integrals investigated by Jaffe et al. [17] and by Arai [1] who have solved the two dimensional WZ model (with space cutoff) by a hamiltonian semigroup approach.

The numerator of (4.10) reads in a more explicit form (we choose for simplicity $\lambda=-1$, but the calculations go through for any $\lambda \in \mathbb{R}$ )

$$
\mathrm{Z}(\mathrm{H})=\left\langle\exp \left(\Omega_{1}-\Omega_{2}+\Omega_{\mathbf{M}}\right) \mid \exp \left(\Gamma_{\mathbf{y}}+\Gamma_{2 a b}\right) \circ \mathrm{H}\right\rangle
$$

The tensor $\Omega=\Omega_{1}-\Omega_{2}+\Omega_{M}$ of the free theory is split into three terms according to the decomposition $\mathrm{K}=\mathrm{K}_{1}-\mathrm{K}_{2}+\mathrm{K}_{\mathrm{M}}$ of the kernel in (3.8) with the bosonic contributions

$\mathrm{K}_{1}=\left[\begin{array}{ll}\mathcal{D}_{1} & 0 \\ 0 & 0\end{array}\right], \quad \mathcal{D}_{1}=\left[\begin{array}{cc}1 & \mathrm{~m} \\ \mathrm{~m} & \mathrm{~m}^{2}\end{array}\right] \mathrm{D}$ and $\mathrm{K}_{2}=\left[\begin{array}{ll}\mathcal{D}_{2} & 0 \\ 0 & 0\end{array}\right], \quad \mathcal{D}_{2}=\left[\begin{array}{ll}0 & 0 \\ 0 & 1\end{array}\right] \delta^{2}(\mathrm{x}-\mathrm{y})$,

and the Majorana contribution $\mathbf{K}_{\mathbf{M}}=\left[\begin{array}{ll}0 & 0 \\ 0 & \mathrm{SC}\end{array}\right]$. 
The interaction tensor (4.7) with UV regularization and volume cut off is split into the

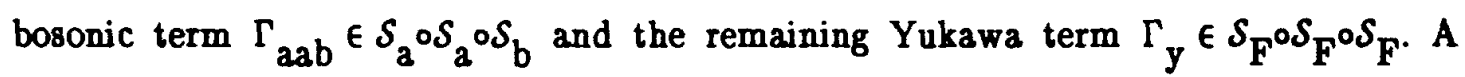
convenient technique to investigate functionals of the type (4.14) is first to evaluate the inner product with respect to the fermionic degrees of freedom, and then to transfer the remaining bosonic part to a functional integral. The first step leads to a product of pfaffians, we shall come back to that step below, but already the free bosonic part causes trouble since the $\delta$-function term $\Omega_{2}$ has the wrong sign. In [32] Nicolai had introduced imaginary auxiliary fields to compensate the minus sign. But that modification is rather unnatural, it yields imaginary mass terms. To simplify the arguments we take the bosonic part of (4.14) alone

$$
\mathrm{Z}_{\mathrm{B}}(\mathrm{H})=\left\langle\exp \left(\Omega_{1}-\Omega_{2}\right)\right| \exp \Gamma_{2 a b} \circ \mathrm{H}>
$$

with $H \in \mathcal{A}\left(\mathcal{S}_{\mathrm{a}} \mathscr{S}_{\mathrm{b}}\right)$. Then the functional is identified with $<\exp \Omega_{1} \mid\left(\exp -\Omega_{2}\right)-\left(\exp \Gamma_{a a b}{ }^{\circ H}\right)>$. The interior product is easily calculated since $\Omega_{2}$ affects only contributions from $S_{b}$ and $\Gamma_{\text {aab }}$ has only a linear dependence on that degree of freedom. As in the case of coherent states, we obtain

$\left(\exp -\Omega_{2}\right)-\exp \Gamma_{\mathrm{aab}}=\exp \left(-\frac{1}{2} \Gamma^{(4)}\right) \circ \exp \Gamma_{\mathrm{aab}}$ with $\left.\Gamma^{(4)}:=\Omega_{2}\right\lrcorner\left(\Gamma_{a a b} \circ \Gamma_{a a b}\right) \in \Lambda_{4}\left(\mathcal{S}_{a}\right)$. In a more pictorial manner we may write $\Gamma^{(4)}=\Gamma_{a a b} \circ \Gamma_{a a b}$ since the contributions from $\mathcal{S}_{\mathrm{b}}$ are totally contracted. The term $\exp \left(-\frac{1}{2} \Gamma^{(4)}\right)$ corresponds to a (nonlocal) $A^{4}$ coupling with the correct minus sign. The calculation of (4.15) leads to

$$
\mathrm{Z}_{B^{(h)}}=\left\langle\exp \Omega_{1}\right| \exp \left(-\frac{1}{2} \Gamma^{(4)}\right) \circ \tilde{\mathrm{H}}>
$$

with a tensor $\tilde{\mathrm{H}} \in \mathcal{A}\left(\mathcal{S}_{\mathrm{B}}\right)$ which is a linear transform of $\mathrm{H}$. For $\mathrm{H} \in \mathcal{\Lambda}\left(\mathcal{S}_{\mathrm{a}}\right)$ it reduces to $\tilde{\mathrm{H}}=\mathrm{H}$, but for $\mathrm{H}=\mathrm{FoG}, \mathrm{F} \in \mathcal{A}\left(\mathcal{S}_{\mathrm{a}}\right)$ and $\mathrm{G} \in \mathcal{A}\left(\mathcal{S}_{\mathrm{b}}\right)$, it includes normal ordering of $\mathrm{G}$ and contractions between $\Gamma_{a a b}$ and $G$. 
The remaining bosonic functional $\left\langle\exp \Omega_{1} \mid \cdot\right\rangle$ is based on a nonnegative quadratic form $<\Omega_{1}\left|f^{*} \circ g\right\rangle=\left(f \mid K_{1} g\right)$ with $f, g \in \mathcal{S}_{B}$. It can therefore be transferred to a gaussian integral. But since the quadratic form is degenerate the measure on $\mathcal{S}_{\mathrm{B}}^{\prime}=\mathcal{S}^{\prime}\left(\mathbb{R}^{2}\right) \bullet \mathrm{C}^{2}$ can be traced back to the usual gaussian measure $\mathrm{d} \mu(\phi)$ on $\mathcal{S}^{\prime}\left(\mathbb{R}^{2}\right)$ with covariance $D(x, y)=\left(m^{2}-\Delta\right)^{-1}(x, y)$. This measure corresponds to a single neutral spin zero boson and exhibits the correct number of degrees of freedom. Tensors $F \in \mathcal{A}_{(\gamma)}\left(\mathcal{S}_{\mathrm{a}}\right)$ or $\mathrm{G} \in \mathcal{A}_{(\gamma)}\left(\mathcal{S}_{\mathrm{b}}\right)$ are mapped onto the entire functions $\eta_{\mathrm{F}}(\mathrm{A})=\langle\exp \mathrm{A} \mid \mathrm{F}\rangle$ with $\mathrm{A} \in \mathcal{S}_{\mathrm{a}}^{\prime}$, or $\eta_{\mathrm{G}}(\mathrm{B})=\langle\exp B \mid G\rangle$ with $\mathrm{B} \in \mathcal{S}_{\mathrm{b}}^{\prime}$. Due to the degeneracy of the form $\left(f \mid K_{1} g\right)$ we can substitute the variables $A(x)=\phi(x) \in \mathcal{S}^{\prime}\left(\mathbb{R}^{2}\right)$ and $B(x)=m \phi(x) \in \mathcal{S}^{\prime}\left(\mathbb{R}^{2}\right)$; and the identification between functionals and integrals is given by $\left\langle\exp \Omega_{1} \mid \mathrm{F} \circ \mathrm{G}\right\rangle=\int \eta_{\mathrm{F}}(\phi) \eta_{\mathrm{G}}(\mathrm{m} \phi) \mathrm{d} \mu(\phi)$. The isomorphism $\mathrm{F} \rightarrow \eta_{\mathrm{F}}(\phi)$ maps the interaction tensor $\Gamma^{(4)}$ onto $\mathrm{K}^{(4)}(\phi)=\frac{1}{4 !}\left\langle\phi^{4} \mid \Gamma^{(4)}\right\rangle=\int \sigma_{\rho}\left(\mathrm{x}_{1}, \mathrm{x}_{2}, \mathrm{x}_{5}\right) \sigma_{\rho}\left(\mathrm{x}_{3}, \mathrm{x}_{4}, \mathrm{x}_{5}\right) \phi\left(\mathrm{x}_{1}\right) \phi\left(\mathrm{x}_{2}\right) \phi\left(\mathrm{x}_{3}\right) \phi\left(\mathrm{x}_{4}\right) \underset{\mathrm{i}}{\prod_{\mathrm{i}}} \mathrm{dx}$

The bosonic functional (4.16) then reads for $H \in \mathcal{A}\left(S_{2}\right)$

$$
Z_{B}(H)=\int e^{-\frac{1}{2} K^{(4)}(\phi)} \eta_{H}(\phi) d \mu(\phi)
$$

The fermionic contribution is still missing. The Yukawa term $\Gamma_{\mathrm{y}} \in \mathcal{S}_{\mathrm{a}} \circ \mathcal{S}_{\mathrm{F}} \circ \mathcal{S}_{\mathrm{F}}$ corresponds to a family of tensors $\Omega^{\mathrm{A}} \in \mathcal{S}_{F^{\circ S_{F}}}$, A $\in \mathcal{S}_{\mathrm{a}}^{\prime}$, such that $\left\langle\chi_{(1)}{ }^{\circ \chi_{(2)}}\right| \Omega^{\mathrm{A}}>=$

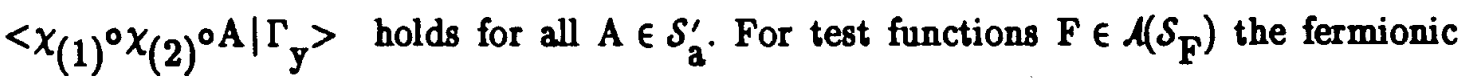
contribution is then given by

$$
\gamma_{F}(A)=\left\langle\operatorname{expA} \circ \exp \Omega_{M}\right| \exp \Gamma_{y} \circ F>=\left\langle\exp \Omega_{M}\right| \exp \Omega^{A} \circ F>
$$

This expression is further evaluated in Appendix $\mathrm{C}$ in terms of pfaffians. We would like to emphasize that there is no need for euclidean Majorana field operators for this calculation. For $\mathrm{H}=\mathrm{FoG}$ with $\mathrm{F} \in \mathcal{A}\left(\mathcal{S}_{\mathrm{F}}\right)$ and $\mathrm{G} \in \mathcal{A}\left(\mathcal{S}_{\mathrm{a}}\right)$ the functional (4.14) is then identified with the integral 


$$
Z(H)=\int e^{-\frac{1}{2} K^{(4)}(\phi)} \gamma_{F}(\phi) \eta_{G}(\phi) d \mu(\phi)
$$

As already mentioned in the calculation of (4.16) the bosonic polynomial $\eta_{\mathrm{G}}(\phi)$ is modified to a more complicated polynomial if $G \in \mathcal{\Lambda}\left(S_{a} \bullet S_{b}\right)$. Integrals of the type (4.19) have been investigated by Jaffe et al. [17] and by Arai [1]. These authors have also removed the UV regularization. In this step the supersymmetric cancellation of singularities plays an essential rôle.

\section{Appendix A}

The algebra $\mathcal{\Lambda}(\mathcal{S})$ is isomorphic to the tensor product $\mathcal{l}^{+}\left(\mathcal{S}_{\bar{\gamma}}\right) \bullet \mathcal{L}\left(\mathcal{S}_{\overline{1}}\right)$ of an algebra of symmetric tensors and an algebra of antisymmetric tensors (Grassmann algebra). Such infinite dimensional algebras have been investigated in great detail by Krée [21]. Proofs with simpler techniques have been given for the symmetric tensor algebra, see e.g. [13]. Nevertheless we shall present here a selfconsistent proof for the nuclearity of the topology of $\ell_{(\gamma)}(\mathcal{S})$ and for the continuity of the graded tensor product in this topology.

The operators $\mathrm{W}^{-\mathrm{p}}, \mathrm{p}=1,2, \ldots$, with $\mathrm{W}$ defined in (2.2) are positive bounded operators on $\boldsymbol{X}$ with a pure point spectrum with finite multiplicity. Moreover already $W^{-1}$ is a HS operator [13]. The second quantization operators $\Gamma\left(W^{-p}\right)$ are then also positive bounded operators with a pure point spectrum on any of the Fock spaces $\mathcal{F}(\gamma)(\mathcal{X}), \gamma \in R$. The spectrum of $\Gamma\left(W^{-p}\right)$ is independent of the value of $\gamma$ which characterizes the norm (2.7). We may therefore take $\mathcal{F}_{(1)}(\mathcal{X})$ to calculate $\operatorname{Tr} \Gamma\left(W^{-2}\right)$. The Fock space $\mathcal{F}_{(1)}(x)$ is isomorphic to $\mathcal{F}_{(1)}^{+}\left(x_{\overline{0}}\right) \bullet \mathcal{F}_{(1)}^{-}\left(x_{T}\right)$, the closure of $\mathcal{l}^{+}\left(x_{\bar{\sigma}}\right) \bullet$ $\mathcal{F}\left(x_{\mathrm{T}}\right)$ with the norm $\|\cdot\|_{1}$. Let $\mathrm{w}_{\mathrm{k}}$ be the restriction of $\mathrm{w}$ to the subspace $\boldsymbol{x}_{\bar{k}}$, $k=0,1$, then the isomorphism of the Fock spaces maps $\Gamma\left(W^{-p}\right)$ onto $\Gamma_{+}\left(W_{0}^{-p}\right) \bullet$ $\Gamma_{\_}\left(W_{1}^{-P}\right)$. Here $\Gamma_{\epsilon}, \epsilon= \pm 1$, indicates the second quantization on the restricted Fock spaces $\mathcal{F}_{(1)}^{+}\left(\mathcal{X}_{\overline{0}}\right)$ of symmetric tensors and $\mathcal{F}_{(1)}\left(\mathcal{X}_{\overline{1}}\right)$ of antisymmetric tensors. The 
factorization $\operatorname{Tr} \Gamma\left(W^{-2}\right)=\operatorname{Tr}_{0} \Gamma_{+}\left(W_{0}^{-2}\right) \cdot \operatorname{Tr}_{1} \Gamma_{-}\left(W_{1}^{-2}\right)$ is obvious and the trace reduces to the well known traces of the grand canonical ensembles

$\operatorname{Tr}_{0} \Gamma_{+}\left(W_{0}^{-2}\right)=\operatorname{det}_{\chi_{\overline{0}}}\left(I-W_{0}^{-2}\right)^{-1} \quad$ and $\operatorname{Tr}_{1} \Gamma_{-}\left(W_{1}^{-2}\right)=\operatorname{det}_{\chi_{I}}\left(I-W_{1}^{-2}\right)$.

Both determinants are finite since

$$
\operatorname{Tr}_{\boldsymbol{X}_{\delta}} \mathrm{W}_{0}^{-2}+\operatorname{Tr}_{\boldsymbol{\chi}_{\mathrm{T}}} \mathrm{W}_{1}^{-2}=\mathrm{Tr}_{\boldsymbol{\chi}} \mathrm{W}^{-2}<\infty \text { and } \mathrm{W}_{0}^{-2} \leq \frac{1}{4}
$$

As a consequence the countable family of norms

$$
\|\mathrm{F}\|_{\gamma, \mathrm{p}}:=\left\|\Gamma\left(\mathrm{W}^{\mathrm{p}}\right) \mathrm{F}\right\|_{\gamma}
$$

with $p \in \mathbb{N}_{0}=\{0,1,2, \ldots\}$ defines a nuclear topology on the intersection $\mathcal{A}_{(\gamma)}(\mathcal{S})=\prod_{p=0}^{\infty} \mathcal{F}(\gamma)\left(x_{p}\right)$, see $[11]$ or $[13]$

The lower bound $W \geq 2$ yields for any $F_{n} \in \lambda_{n}(S)$ the estimate

$$
\left\|F_{n}\right\|_{\gamma, p+q}=\left\|\Gamma\left(W^{p+q}\right) F_{n}\right\|_{\gamma} \geq 2^{n q} \| \Gamma\left(W^{p_{F}}\left\|_{n}=2^{n q}\right\| F_{n} \|_{\gamma, p} .\right.
$$

For $F_{m} \in \mathcal{A}_{m}(\mathcal{S})$ and $G_{n} \in \mathcal{l}_{n}(\mathcal{S})$ we have from (2.6) and (2.7)

$$
\begin{gathered}
\left\|F_{m} \circ G_{n}\right\|_{\gamma, p}^{2} \leq\left[\frac{(m+n) !}{(m ! n !}\right]^{\gamma+1}\left\|F_{m}\right\|_{\gamma, p}^{2} \cdot\left\|G_{n}\right\|_{\gamma, p}^{2} \\
\leq 2^{(\gamma+1)(m+n)}\left\|F_{m}\right\|_{\gamma, p}^{2} \cdot\left\|G_{n}\right\|_{\gamma, p}^{2}
\end{gathered}
$$

Now take $F=\underset{m}{\sum} F_{m}$ and $G=\underset{n}{\sum} G_{n}$ with $F_{n}, G_{n} \in \mathcal{A}_{n}(S)$, then (2.6), (2.7) and (A.3) yield 


$$
\begin{aligned}
& \|\mathrm{F} \circ \mathrm{G}\|_{\gamma, \mathrm{p}}^{2}=\underset{\mathrm{N}}{\Sigma}\left\|\underset{\mathrm{m}+\mathrm{n}=\mathrm{N}}{\Sigma} \mathrm{F}_{\mathrm{m}} \circ \mathrm{G}_{\mathrm{n}}\right\|_{\gamma, \mathrm{p}}^{2} \\
& \leq \sum_{N}\left[\sum_{m+n=N} 2^{2^{\frac{1}{2}(\gamma+1)(m+n)}}\left\|F_{m}\right\|_{\gamma, p} \cdot\left\|G_{n}\right\|_{\gamma, p}\right]^{2} \\
& \leq \sum_{m, n}(1+m+n) 2^{(\gamma+1)(m+n)}\left\|F_{m}\right\|_{\gamma, p}^{2} \cdot\left\|G_{n}\right\|_{\gamma, p}^{2}
\end{aligned}
$$

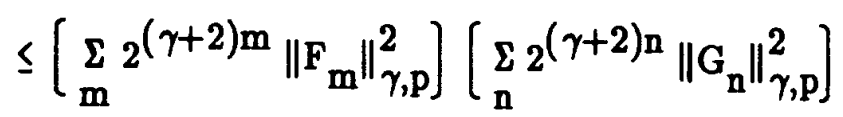

$$
\begin{aligned}
& \stackrel{(\mathrm{A.2})}{\leq}\|\mathrm{F}\|_{\gamma, \mathrm{p}+\mathrm{q}}^{2} \cdot\|\mathrm{G}\|_{\gamma, \mathrm{p}+\mathrm{q}}^{2} \text { if } \mathrm{q} \geq \gamma+2 .
\end{aligned}
$$

The tensor product is therefore a continuous bilinear mapping $\lambda_{(\gamma)}(\mathcal{S}) \times \mathcal{A}_{(\gamma)}(\mathcal{S}) \rightarrow$ $\mathcal{A}_{(\gamma)}(\mathcal{S})$ for any $\gamma \in \mathbb{R}$.

\section{Appendix B}

In this Appendix some results about the bilinear forms of the two-point Schwinger functions for charged and neutral bosons or spin- $\frac{1}{2}$ fermions are presented with special emphasis on Majorana fermions. Following [25] we give a construction of the bilinear form for neutral particles from that of charged particles. In the context of supersymmetry the theory for bosons and fermions is developed in a parallel way. The final part of this Appendix is deroted to physical (OS) positivity.

For a quantum field theory of charged bosons/fermions with $\mathrm{n}$-component field operators $\phi_{\alpha}(\underline{x}), \phi_{\alpha}^{*}(\underline{x}), \alpha=1, \ldots, n$, defined on a Minkowski space with signature $(d-1,1)$, i.e. $\underline{x}=(\vec{x}, c t) \in R^{d-1} \times R$, the kernel of the bilinear form of the two-point Schwinger functions is calculated by analytic continuation of the $2 \mathrm{n} \times 2 \mathrm{n}$ matrix 


$$
\left[\begin{array}{lll}
\left\langle\operatorname{vac}\left|\phi^{*}(\underline{x}) \phi^{*}(\mathbf{y})\right| \operatorname{vac}\right\rangle & \left.<\operatorname{vac}\left|\phi^{*}(\underline{x}) \phi(\underline{y})\right| \operatorname{vac}\right\rangle \\
\langle\operatorname{vac}| \phi(\underline{x}) & \phi^{*}(\underline{y})|\operatorname{vac}\rangle & <\operatorname{vac}|\phi(\underline{x}) \phi(y)| \operatorname{vac}\rangle
\end{array}\right]
$$

Here $\phi_{\alpha}^{*}$ indicates the adjoint operator $\phi_{\alpha}^{+}$for bosons, and $\phi_{\alpha}^{*}=\underset{\beta}{\Sigma} \phi_{\beta}^{+}\left(\gamma_{d}\right)_{\beta \alpha}$ for Dirac spinors. The bilinear symmetric/antisymmetric form for bosons $(\sigma=+1) /$ fermions $(\sigma=-1)$ then reads as

$$
\omega_{\sigma}(f, g)=\int f^{T}(x)\left[\begin{array}{cc}
0 & \sigma S_{\sigma}^{T}(y, x) \\
S_{\sigma}(x, y) & 0
\end{array}\right] g(y) d x d y
$$

with test functions $\mathrm{f}, \mathrm{g} \in \mathcal{S}=\mathcal{S}\left(\mathbb{R}^{\mathrm{d}}\right) \bullet \mathrm{c}^{2 \mathrm{n}}$ which are represented as column vectors. For a free spin- 0 field we have $n=1$ and $S_{+}(x, y)$ is the inverse Klein-Gordon operator $D(x, y)=\left(m^{2}-\Delta\right)^{-1}(x, y) ;$ for free Dirac fermions $S_{-}(x, y)$ is the inverse euclidean Dirac operator $(m-g)^{-1}(x, y)$. In Sect. 3 . the bosonic sector has been extended by auxiliary fields. Then the basic Schwinger function gets more intricate. In the case of the charged WZ model in two dimensions the bilinear form has still the structure (B.2) but with $S_{+}(x, y)=D(x, y)$ as defined in (3.9). The form (B.2) allows for a natural isotropic decomposition of the test function space: $S=\mathcal{E} \bullet \mathcal{E}^{*}$ with $\mathcal{E} \simeq \mathcal{E}^{*} \simeq \mathcal{S}\left(\mathbf{R}^{\mathrm{d}}\right) \bullet \mathrm{C}^{\mathrm{n}}$, such that $f \in \mathcal{S}$ is represented by $f=\left[\begin{array}{l}a \\ b\end{array}\right]$ with $a \in \mathcal{E}$ and $b \in \mathcal{E}^{*}$. The involution (2.3) is then

$$
f^{*}(x)=\left[\begin{array}{ll}
0 & 1 \\
1 & 0
\end{array}\right] f(x)
$$

with the $\mathrm{n} \times \mathrm{n}$ identity matrix $\mathbf{1}_{\mathrm{n}}$. In all cases for which a linear local charge conjugation of the field operators in (B.1) exists there is a linear relation between the Wightman functions which extends to the Schwinger functions

$$
S_{\sigma}(x, y) C_{\sigma}=\sigma C_{\sigma}^{T} S_{\sigma}^{T}(y, x)
$$


This identity together with $\mathrm{C}_{\sigma}^{\mathrm{T}}=\mathrm{C}_{\sigma}^{-1}$ actually determines the charge conjugation matrices up to a sign abiguity. For bosons we obtain $C_{+}=1_{n}$, and for Dirac spinors $C_{-}$ is the charge conjugation matrix defined by (1.1) which only exists for $d=2,3,4 \bmod 8$ dimensions [33] [25]. The charge conjugation property (B.4) corresponds to a linear charge conjugation operator $U$ on the test function space

$$
\mathrm{Uf}=\left[\begin{array}{cc}
0 & \mathrm{C} \\
\mathrm{C}^{-1} & 0
\end{array}\right] \mathrm{f}
$$

such that

$$
w(\mathrm{Uf}, \mathrm{Ug})=w(f, g)
$$

Here and in the following equations we omit the subscript $\sigma$. The property $\mathrm{U}^{2}=$ id yields that

$$
P=\frac{1}{2}(i d+U)=\frac{1}{2}\left[\begin{array}{ll}
1 & C \\
C^{-1} & 1
\end{array}\right]
$$

is a projection operator. The form

$$
w(P f, P g)=\frac{1}{2} \int f^{T}(x)\left[\begin{array}{ll}
C S & \sigma S^{T} \\
S & S C
\end{array}\right] g(y) d x d y
$$

is then the (degenerate) bilinear form for neutral particles. This identification can be directly checked with (B.1). Actually there are two forms related to the charge conjugation matrices $\pm \mathrm{C}$. The degrees of freedom for a charged particle/antiparticle pair correspond to the degrees of freedom of two neutral particles [25]. The form (B.8) has as kernel the kernel of the projection operator $P$ $\boldsymbol{X}=\operatorname{Ker} P=\left\{\left[\begin{array}{c}-\mathrm{Cb} \\ \mathrm{b}\end{array}\right] \mid \mathrm{b} \in \mathcal{E}^{*}\right\}$. The test function space for neutral fields is therefore 
the factor space $\boldsymbol{x}^{0}=\boldsymbol{X} / \boldsymbol{X}$ which for technical convenience will be identified with $\mathcal{E}^{*}$. The surjective mapping $\Lambda$ from $x$ onto $\boldsymbol{x}^{0} \simeq \mathcal{E}^{*}$ is then given by

$$
\Lambda\left[\begin{array}{l}
a \\
b
\end{array}\right]=C^{-1} a+b
$$

The nondegenerate bilinear form for neutral particles $\omega^{0}: \mathcal{E}^{*} \times \mathcal{E}^{*} \rightarrow \mathrm{C}$ has to satisfy $\omega^{0}\left(\Lambda f_{1}, \Lambda f_{2}\right)=2 \omega\left(\mathrm{Pf}_{1}, \mathrm{Pf}_{2}\right)$. Its computation leads to

$$
\omega^{0}\left(b_{1}, b_{2}\right)=\int b_{1}^{T}(x) S(x, y) C b_{2}(y) d x d y
$$

For a single neutral spin -0 particle this form coincides with $\int b_{1}(x) D(x, y) b_{2}(y) d x d y$, for Majorana fermions we obtain (3.7). The kernel of (B.10) is exactly the analytic continuation of $\langle\operatorname{vac}|\phi(\underline{x}) \phi(y)| \operatorname{vac}\rangle$.

To discuss physical (OS) positivity we need the reflection of the "time" coordinate $\vartheta x=v\left(\vec{x}, x_{d}\right)=\left(\vec{x},-x_{d}\right), \vec{x} \in \mathbb{R}^{d-1}$. Then the antilinear OS involution is defined for test functions of charged particles by $[34,36,10]$

$$
(T f)(x)=\left[\begin{array}{ll}
0 & R \\
R^{T} & 0
\end{array}\right] f(\mathscr{F})
$$

with $R=1_{n}$ for bosons and $R=\gamma_{d}$ for Dirac fermions. The bilinear form (B.2) is

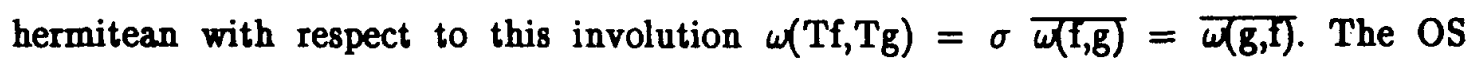
sesquilinear form is calculated as

$$
\omega(T f, g)=\int f^{+}(x)\left[\begin{array}{cc}
R S(\partial x, y) & T^{T} S^{T} \\
0 & \left.\sigma R^{2}, \partial x\right)
\end{array}\right] g(y) d x d y
$$

The properties of the Klein-Gordon and of the Dirac operators yield then OS positivity 
$\omega(T f, f) \geq 0 \quad$ if $\operatorname{supp} f \in \mathbb{R}^{d-1} \times \mathbb{R}_{+}$.

The transition to neutral particles is again given by the surjective mapping (B.9). We define the OS involution on $\boldsymbol{x}^{0}$ such that $\mathrm{T}^{0} \Lambda=\Lambda \mathrm{T}$. With the identification $\boldsymbol{x}^{0}=\mathcal{E}^{*}$ this antilinear operator is then calculated as

$$
\left(T^{0} b\right)(x)=R^{T} C b(\delta x)= \begin{cases}b(\delta x) & \text { for bosons, } \\ \gamma_{d}^{T} C_{-} \delta(\delta x) & \text { for Majorana fermions. }\end{cases}
$$

The positivity $\omega^{0}\left(T^{0} b, b\right) \geq 0$ if supp $b \subset R^{d-1} \times R_{+}$is then a consequence of (B.13) or it may be directly checked with the form (B.10). This result is sufficient for the OS positivity of the form (3.8) of the WZ model since the matrix $\left[\begin{array}{cc}1 & m \\ m & m^{2}\end{array}\right]$ is nonnegative and the $\delta$-function term does not contribute to the OS-form due to the restriction of the supports of the test functions.

Appendix C

Calculations for euclidean Majorana fermions are mainly performed with euclidean field operators $[32,3]$. In this Appendix we give the essential identities for a functional approach which has been used for Dirac and Weyl fermions in [22]. The results for Majorana fermions have first been presented by Jaffe et al. in the language of pfaffians [18]. These authors have also investigated the regularization of pfaffians, a problem which we will not discuss here.

The antisymmetric tensor product is written as FoG and defined by (2.6) with a modified projection $P_{m+n}$ onto the antisymmetric tensors only. Let $\langle f \mid g\rangle=\left(f^{*} \mid g\right)$ be the bilinear pairing on $x$ derived from the involution $f^{*}(\alpha, x)=f(\alpha, x)$. Then there is an isomorphism between skew symmetric operators $\mathrm{K}: \boldsymbol{x} \rightarrow \boldsymbol{x},\langle\mathrm{f} \mid \mathrm{Kg}\rangle=-\langle\boldsymbol{g} \mid \mathrm{Kf}\rangle$, and tensors $\Omega \in \mathcal{I}_{2}\left(\mathcal{S}^{\prime}\right)$ given by

$$
\langle\Omega(\mathrm{K}) \mid \mathrm{f \circ g}\rangle=\langle\mathrm{f} \mid \mathrm{Kg}\rangle, \quad \mathrm{f}, \mathrm{g} \in \mathcal{S} .
$$


Then the basic Lemma for the calculations is:

Let $\mathrm{K}$ and $\mathrm{L}$ be bounded skew symmetric operators on $\boldsymbol{X}$ such that KL is traceclass, then the following identity holds for all $\mathrm{z} \in \mathrm{C}$ and all $\mathrm{F} \in \mathcal{L}(\mathcal{S})$

$$
\langle\exp \Omega(\mathrm{K}) \mid \exp \Omega(\mathrm{zL}) \circ \mathrm{F}\rangle=\sqrt{\operatorname{det}(I-\mathbf{K L L})}\left\langle\exp \Omega\left(\mathrm{M}_{\mathbf{z}}\right) \mid \mathrm{F}\right\rangle
$$

with $M_{z}=K(I-z L K)^{-1}=(I-z K L)^{-1} K$. Moreover (C.2) is an extire analytic function in $\mathbf{z}$.

The proof of this Lemma follows from Sect. IV of [18]; or it may be derived with the techniques of [22] where this identity has been given for a specific type of skew symmetric operators (related to Dirac fields). The square root of the determinant is the relative pfaffian $p f(K, z L)=\sqrt{\operatorname{det}(I-z K L)}$ of $[18]$. For $F=f_{1} \circ \ldots \circ f_{2 n}, f_{i} \in X$, the whole expression (C.2) is the pfaffian minor $\mathrm{pf}\left(\mathrm{K}, \mathrm{L} ; \mathrm{f}_{1}, \ldots, \mathrm{f}_{2 \mathrm{n}}\right)$ of $[17]$. The relative pfaffian $\mathrm{pf}(\mathrm{K}, \mathrm{zL})$ is an entire analytic function in $\mathrm{z} \in \mathrm{C}$. Its zeros compensate the poles of the factor $<\exp \Omega\left(M_{z}\right)\left|f_{1} \circ \ldots \circ f_{2 n}\right\rangle=p f\left(\left\langle f_{i} \mid M_{z} f_{j}\right\rangle\right)$.

As application we consider a Majorana fermion in a (nonlocal) potential V. We assume that $V$ has already been sufficiently regularized such that $(m-2)^{-1} V$ is a traceclass operator. Moreover CV with the charge conjugation matrix (1.1) has to be a bounded skew symmetric operator on $X$, e.g. for any fixed $A \in \mathcal{S}_{\mathrm{a}}^{\prime}$ the regularized Yukawa term in (4.7) is of this form. The tensor $\Omega_{M}$ of a free Majorana theory is exactly given by $\Omega(S C)$ with $S=(m-\not)^{-1}$, see (3.7). Now take $K=S C$ and $L=C V$, then (C.2) reads

$$
<\exp \Omega(\mathrm{SC})\left|\exp \Omega(\mathrm{CV}) \circ \mathrm{F}>=\sqrt{\operatorname{det}(I-S V)}<\exp \Omega\left(\mathrm{S}_{\mathrm{V}} \mathrm{C}\right)\right| \mathrm{F}>
$$


where $S_{V}=(m-\partial-V)^{-1}$ is the inverse Dirac operator with the potential V. For $F=f_{1} \circ \ldots \circ f_{2 n}$ the last term is the pfaffian of the skew symmetric $2 n \times 2 n$ matrix $\left(<f_{i}\left|S_{V} C_{j}\right\rangle\right)$. The identity (C.3) is the Matthews-Salam formula [30] for euclidean Majorana fermions. The functionals for Dirac fermions can be obtained from (C.2) if one choses the kernel of (3.6) as the skew symmetric operator $K$, see e.g. [22].

\section{Acknowledgements}

This paper is supported by a German-Polish research program (WTZ mit Polen, Projekt Nr. X 081.13) which allowed Z.H. to visit Kaiserslautern and J.K. to visit Wroclaw. 
References

[1] A. Arai, "A general class of infinite dimensional Dirac operaters and path integral representation of their index." J. Funct. Anal. 105 (1992), 342-408.

[2] H. J. Borchers, "On structure of the algebra of field operators." Nuovo Cimento 24 (1962), 214-236.

[3] D. Borthwick, "Euclidean Majorana fermions, fermionic integrals and relative pfaffians." J. Math. Phys. 34 (1993), 2691-2712.

[4] N. Bourbaki, Elements of Mathematics: Algebra I, chapt. 1-9. Paris: Hermann 1974.

[5] S. Browne, "Spontaneous breakdown and finiteness of supersymmetric theories in two dimensions." Phys. Lett. 59B (1975), 253-255.

[6] E. R. Caianiello, Combinatorics and renormalization in quantum field theory. Reading: W. A. Benjamin 1973.

[7] B. DeWitt, Supermanifolds. Cambridge: CUP 1984.

[8] J. P. Eckmann and H. Epstein, "Time-ordered products and Schwinger functions." Commun. Math. Phys. 64 (1979), 95-130.

[9] H.M. Fried, Functional Methods and Models in Quantum Field theory. Cambridge: MIT Press 1972.

[10] J. Fröhlich and K. Osterwalder, "Is there a euclidean field theory for fermions?" Helv. Phys. Acta 47 (1974), 781-805.

[11] I.M. Gelfand and N.Ya. Vilenkin, Generalized Functions, Vol. IV, New York: Academic Press 1964.

[12] J. Glimm and A. Jaffe, Quantum Physics. A functional integral point of view. N. Y. : Springer 1987.

[13] T. Hida, H.-H. Kuo, J. Potthoff and L. Streit, White noise, an infinite dimensional calculus. Dortrecht: Kluwer 1993.

[14] J. Iliopoulos and B. Zumino, "Broken supergauge symmetry and renormalization". Nucl. Phys. B76 (1974), 310-332. 
[15] A. Jadczyk and K. Pilch, "Superspaces and supersymmetries." Commun. Math. Phys. 78 (1981), 371-390.

[16] A. Jaffe, A. Lesniewski and J. Weitsman, "The two-dimensional, $N=2$ Wess-Zumino model on a cylinder." Commun. Math. Phys. 114 (1988), $147-165$.

[17] A. Jaffe, A. Lesniewski and J. Weitsman, "The loop space $S^{1} \rightarrow R$ and supersymmetric quantum fields." Ann. Phys. 183 (1988), 337-351.

[18] A. Jaffe, A. Lesniewski and J. Weitsman, "Pfaffians on Hilbert space." J. Funct. Annal. 83 (1989), 348-363.

[19] A. Jaffe and A. Lesniewski, "Geometry of supersymmetry." In: G. Velo and A. S. Wightman (eds.): Constructive quantum field theory II. New-York: Plenum $1990,283-305$.

[20] A. Yu. Khrennikov, "Functional superanalysis." Russ. Math. Surveys 43 (1988), $103-137$.

[21] P. Krée, "Formes et coformes sur un espace nucléaire complet." In: Seminaire Paul Krée, Se année: 1976/77. Paris: Secrétariat mathematique, 1978.

[22] J. Kupsch, "Functional integration for euclidean Fermi fields." In: $M$. Pawlowski and R. Raczka (eds.): Gauge theories of fundamental interactions. Singapore: World Scientific 1990, 425-451.

[23] J. Kupsch, "A probabilistic formulation of bosonic and fermionic integration." Rev. Math. Phys. 2 (1990), 457-477.

[24] J. Kupsch, "Non-commutative integration and euclidean quantum fields for fermions". In: J.Q. Liang et al. (eds.): Proceedings of international symposium on advanced topics of quantum physics - Shanzi University 1992. Beijing: Science Press 1993, 294-304.

[25] J. Kupsch and W. D. Thacker, "Euclidean Majorana and Weyl spinors." Fortschr. Phys. 38 (1990), 35-62.

[26] D. A. Leites, "Introduction to the theory of supermanifolds." Russ. Math. Surveys 35 (1980), $1-64$. 
[27] J. Lukierski, "Euclidean superalgebras for $3 \leq \mathrm{D} \leq 10$." In: G. W. Gibbons, S. W. Hawking and P. K. Townsend (eds.): Supersymmetry and its applications: Superstrings, anomalies and supergravity. Cambridge: CUP 1986, 463-481.

[28] J. Lukierski and A. Nowicki, "On superfield formulation of euclidean supersymmetry." J. Math. Phys. 25 (1984), 2545-2549.

[29] I. Martin-Hernandez and J. G. Taylor, "On euclidean supersymmetry." Phys. Lett. 185B (1987), 99-103.

[30] P.T. Matthews and A. Salam, "Propagators of quantized fields." Nuovo Cimento 2 (1955), 120-134.

[31] H.J.W. Müller-Kirsten and A. Wiedemann, "Dirac quantization of a supersymmetric field theory." Z. Phys. C 35 (1987), 471-478.

[32] H. Nicolai, "A possible constructive approach to (super- $\left.\phi^{3}\right)_{4}$ " I, Nucl. Phys. B140 (1978), 294-300; II and III, Nucl. Phys. B156 (1979), 157-176 and 177-188.

[33] P. van Nieuwenhuizen, "Six lectures at the Trieste 1981 summerschool on supergravity." In: S. Ferrara and J. G. Taylor (eds.), Supergravity '81. Cambridge: CUP 1982.

[34] K. Osterwalder and R. Schrader, "Axioms for Euclidean Green's functions. I," Commun. Math. Phys. 31 (1973), 83-112.

[35] K. Osterwalder and R. Schrader, "Axioms for Euclidean Green's functions. II," Commun. Math. Phys. 42 (1975), 281-305.

[36] K. Osterwalder and R. Schrader, "Euclidean Fermi fields and a Feynman-Kac formula for boson-fermion models." Helv. Phys. Acta 42 (1973), 277-302.

[37] O. Piguet and K. Sibold, Renormalized supersymmetry. Boston: Birkhäuser 1986 .

[38] A. Rogers, "A global theory of supermanifolds." J. Math. Phys. 21 (1980), 1352-1365.

[39] A. Rogers, "Consistent superspace integration." J. Math. Phys. 26 (1985), 385-392. 
[40] A. Salam and J. Strathdee, "Super-gauge transformations." Nucl. Phys. B76 (1974), 477-482.

[41] M. Scheunert, The theory of Lie superalgebras. (Lect. Notes in Math. 716). Berlin/ Heidelberg/ N.Y.: Springer 1979.

[42] O. G. Smolyanov and E. T. Shavgulidze, "The Fourier transform and pseudodifferential operators in superanalysis." Sov. Math. Dokl. 37 (1988), $476-481$.

[43] M. F. Sohnius, "Introducing supersymmetry." Phys. Rep. 128 (1985), 39-204.

[44] V. S. Vladimirov and I. V. Volovich, "On the definition of the integral in superspace." Sov. Math. Dokl. 35 (1985), 817-819.

[45] J. Wess and B. Zumino, "Supergauge transformations in four dimensions." Nucl. Phys. B70 (1974), 39-50.

[46] J. Wess and B. Zumino, "A Lagrangian model invariant under supergauge transformations." Phys. Lett. 49B (1974), 52-54.

[47] B. Zumino, "Relativistic strings and supergauges." In: E. R. Caianiello (ed.), Renormalization and invariance in quantum field theory. N. Y.: Plenum 1974, $367-381$.

[48] B. Zumino, "Euclidean supersymmetry and the many-instanton problem." Phys. Lett. 69B (1977), 369-371. 
